\title{
Dielectric elastomer composites: A general closed-form solution in the small-deformation limit
}

\author{
Stephen A. Spinelli, Victor Lefèvre, Oscar Lopez-Pamies \\ Department of Civil and Environmental Engineering, University of Illinois, Urbana-Champaign, IL 61801, USA
}

\begin{abstract}
A solution for the overall electromechanical response of two-phase dielectric elastomer composites with (random or periodic) particulate microstructures is derived in the classical limit of small deformations and moderate electric fields. In this limit, the overall electromechanical response is characterized by three effective tensors: a fourth-order tensor describing the elasticity of the material, a second-order tensor describing its permittivity, and a fourth-order tensor describing its electrostrictive response. Closed-form formulas are derived for these effective tensors directly in terms of the corresponding tensors describing the electromechanical response of the underlying matrix and the particles, and the one- and two-point correlation functions describing the microstructure. This is accomplished by specializing a new iterative homogenization theory in finite electroelastostatics (Lopez-Pamies, 2014) to the case of elastic dielectrics with even coupling between the mechanical and electric fields and, subsequently, carrying out the pertinent asymptotic analysis.

Additionally, with the aim of gaining physical insight into the proposed solution and shedding light on recently reported experiments, specific results are examined and compared with an available analytical solution and with new full-field simulations for the special case of dielectric elastomers filled with isotropic distributions of spherical particles with various elastic dielectric properties, including stiff high-permittivity particles, liquid-like high-permittivity particles, and vacuous pores.
\end{abstract}

Key words: Electrostriction; Electroactive materials; Microstructures; Iterated homogenization

\section{Introduction}

The object of this paper is to generate a rigorous analytical solution for the overall or macroscopic electromechanical response of dielectric elastomer composites with (random or periodic) particulate microstructures in the limit of small deformations and moderate electric fields. At a microscopic level, a dielectric elastomer composite is taken to consist of a statistically uniform distribution of particles bonded perfectly to a continuous matrix phase. The domain occupied by the entire composite in its (undeformed and stress-free) ground state is denoted by $\Omega$. We assume that the characteristic length scale of the underlying particles is much smaller than the size of $\Omega$ and, for convenience, choose units of length so that $\Omega$ has unit volume. The constitutive behaviors of the matrix $(r=1)$ and the particles $(r=2)$ are characterized by "total" free energies $W^{(r)}$ that are objective functions of the deformation gradient tensor $\mathbf{F}$ and objective and even functions of the Lagrangian electric field $\mathbf{E}$ so that $W^{(r)}(\mathbf{Q F}, \mathbf{E})=W^{(r)}(\mathbf{F}, \mathbf{E})=W^{(r)}(\mathbf{F},-\mathbf{E})$ for all $\mathbf{Q} \in$ Orth+. At each material point $\mathbf{X} \in \Omega$, the first Piola-Kirchhoff stress tensor $\mathbf{S}$ and the Lagrangian electric displacement field D are hence given by (Dorfmann and Ogden, 2005; see also Suo et al., 2008)

$$
\mathbf{S}=\frac{\partial W}{\partial \mathbf{F}}(\mathbf{X}, \mathbf{F}, \mathbf{E}) \quad \text { and } \quad \mathbf{D}=-\frac{\partial W}{\partial \mathbf{E}}(\mathbf{X}, \mathbf{F}, \mathbf{E})
$$

Email addresses: sspinel2@illinois.edu (Stephen A. Spinelli), vlefevre@illinois.edu (Victor Lefèvre), pamies@illinois.edu (Oscar Lopez-Pamies) 
with

$$
W(\mathbf{X}, \mathbf{F}, \mathbf{E})=\left[1-\theta^{(2)}(\mathbf{X})\right] W^{(1)}(\mathbf{F}, \mathbf{E})+\theta^{(2)}(\mathbf{X}) W^{(2)}(\mathbf{F}, \mathbf{E}),
$$

where $\theta^{(2)}(\mathbf{X})$ stands for the indicator function of the regions occupied by the particles: $\theta^{(2)}(\mathbf{X})=1$ if $\mathbf{X}$ is inside a particle and $\theta^{(2)}(\mathbf{X})=0$ otherwise.

For random microstructures, the indicator function $\theta^{(2)}$ in $(2)$ is known only in a probabilistic sense. For periodic microstructures, on the other hand, $\theta^{(2)}$ is completely known once a unit cell and the lattice over which it is repeated are specified. In either case, at any rate, we shall require but partial knowledge of $\theta^{(2)}$ in terms of the one- and two-point correlation functions. In view of the assumed statistical uniformity of the microstructure, these functions are insensitive to translations and thus given by (see, e.g., Chapter 15 in Milton, 2002 and references therein)

$$
p^{(2)}=\int_{\Omega} \theta^{(2)}(\mathbf{X}) \mathrm{d} \mathbf{X} \quad \text { and } \quad p^{(22)}(\mathbf{Y})=\int_{\Omega} \theta^{(2)}(\mathbf{Y}+\mathbf{X}) \theta^{(2)}(\mathbf{X}) \mathrm{d} \mathbf{X} .
$$

Geometrically, the one-point correlation function $p^{(2)}$ represents the probability that a point lands in a particle when it is dropped randomly in $\Omega$. In other words, $p^{(2)}$ is nothing more than the volume fraction of particles in the undeformed configuration; henceforth, we shall utilize the standard notation $p^{(2)}=c$. The two-point correlation function $p^{(22)}$ represents the probability that the ends of a rod of length and orientation described by the vector $\mathbf{Y}$ land within (the same or two different) particles when dropped randomly in $\Omega$. Accordingly, $p^{(22)}$ contains finer information about the size, shape, and spatial distribution of the particles in the undeformed configuration.

Granted the statistical uniformity of the microstructure and the separation between microscopic and macroscopic length scales, the overall constitutive response for the composite is defined as the relation between the volume averages of the first Piola-Kirchhoff stress $\overline{\mathbf{S}} \doteq \int_{\Omega} \mathbf{S}(\mathbf{X}) \mathrm{d} \mathbf{X}$ and electric displacement $\overline{\mathbf{D}} \doteq \int_{\Omega} \mathbf{D}(\mathbf{X}) \mathrm{d} \mathbf{X}$ and the volume averages of the deformation gradient $\overline{\mathbf{F}} \doteq \int_{\Omega} \mathbf{F}(\mathbf{X}) \mathrm{d} \mathbf{X}$ and electric field $\overline{\mathbf{E}} \doteq \int_{\Omega} \mathbf{E}(\mathbf{X}) \mathrm{d} \mathbf{X}$ over the undeformed configuration $\Omega$ when the composite is subjected to affine boundary conditions. Such a relation can be compactly written as (Lopez-Pamies, 2014)

$$
\overline{\mathbf{S}}=\frac{\partial \bar{W}}{\partial \overline{\mathbf{F}}}(\overline{\mathbf{F}}, \overline{\mathbf{E}}, c) \quad \text { and } \quad \overline{\mathbf{D}}=-\frac{\partial \bar{W}}{\partial \overline{\mathbf{E}}}(\overline{\mathbf{F}}, \overline{\mathbf{E}}, c),
$$

where

$$
\bar{W}(\overline{\mathbf{F}}, \overline{\mathbf{E}}, c)=\min _{\mathbf{F} \in \mathcal{K}} \max _{\mathbf{E} \in \mathcal{E}} \int_{\Omega} W(\mathbf{X}, \mathbf{F}, \mathbf{E}) \mathrm{d} \mathbf{X},
$$

the effective free energy function, corresponds physically to the total electroelastic free energy (per unit undeformed volume) of the composite. In these last expressions, the volume fraction of particles $c$ has been included as an explicit argument in the effective energy $\bar{W}$ for later convenience and $\mathcal{K}, \mathcal{E}$ denote sufficiently large sets of admissible deformation gradients $\mathbf{F}$ and curl-free electric fields $\mathbf{E}$ with prescribed volume averages $\overline{\mathbf{F}}$ and $\overline{\mathbf{E}}$. For later reference, we note that the effective free energy function (5) is, by definition, objective in $\overline{\mathbf{F}}$ and objective and even in $\overline{\mathbf{E}}$, namely, $\bar{W}(\overline{\mathbf{F}}, \overline{\mathbf{E}}, c)=\bar{W}(\overline{\mathbf{Q}} \overline{\mathbf{F}}, \overline{\mathbf{E}}, c)=\bar{W}(\overline{\mathbf{F}},-\overline{\mathbf{E}}, c)$ for all $\overline{\mathbf{Q}} \in$ Orth+, much like its local counterpart (2).

\subsection{The classical limit of small deformations and moderate electric fields}

The focus of this paper is on the classical limit of small macroscopic deformations and moderate macroscopic electric fields. More precisely, defining $\zeta$ as a vanishingly small parameter, the deformation measure $\overline{\mathbf{H}} \doteq \overline{\mathbf{F}}-\mathbf{I}$, with $\mathbf{I}$ denoting the identity in the space of second-order tensors, is assumed to be $O(\zeta)$ while the electric field $\overline{\mathbf{E}}$ is assumed to be $O\left(\zeta^{1 / 2}\right)$ (see, e.g., Stratton, 1941; Toupin, 1956; Tian et al., 2012). In this limit, the effective free energy function (5) takes the asymptotic form

$$
\bar{W}(\overline{\mathbf{F}}, \overline{\mathbf{E}}, c)=\frac{1}{2} \bar{H}_{i j} \widetilde{L}_{i j k l}(c) \bar{H}_{k l}-\frac{1}{2} \bar{E}_{i} \widetilde{\epsilon}_{i j}(c) \bar{E}_{j}+\bar{H}_{i j} \widetilde{M}_{i j k l}(c) \bar{E}_{k} \bar{E}_{l}-\bar{E}_{i} \bar{E}_{j} \widetilde{\tau}_{i j k l}(c) \bar{E}_{k} \bar{E}_{l}+O\left(\zeta^{3}\right),
$$


where $\widetilde{\mathbf{L}}$ stands for the effective modulus of elasticity, $\widetilde{\boldsymbol{\epsilon}}$ denotes the effective permittivity, $\widetilde{\mathbf{M}}$ is the effective electrostrictive tensor, and $\widetilde{\boldsymbol{\tau}}$ represents the effective permittivity of second order. Because of the energy character and overall objectivity of the definition (5), these tensors exhibit the symmetries $\widetilde{L}_{i j k l}=\widetilde{L}_{k l i j}=$ $\widetilde{L}_{j i k l}=\widetilde{L}_{i j l k}, \widetilde{\epsilon}_{i j}=\widetilde{\epsilon}_{j i}, \widetilde{M}_{i j k l}=\widetilde{M}_{j i k l}=\widetilde{M}_{i j l k}, \widetilde{\tau}_{i j k l}=\widetilde{\tau}_{j i k l}=\widetilde{\tau}_{k j i l}=\widetilde{\tau}_{l j k i}=\widetilde{\tau}_{i k j l}=\widetilde{\tau}_{i l k j}=\widetilde{\tau}_{i j l k}$. The corresponding relations for the macroscopic stress and electric displacement (4) reduce to

$$
\bar{S}_{i j}=\frac{\partial \bar{W}}{\partial \bar{F}_{i j}}(\overline{\mathbf{F}}, \overline{\mathbf{E}}, c)=\widetilde{L}_{i j k l}(c) \bar{H}_{k l}+\widetilde{M}_{i j k l}(c) \bar{E}_{k} \bar{E}_{l}+O\left(\zeta^{2}\right)
$$

and

$$
\bar{D}_{i}=-\frac{\partial \bar{W}}{\partial \bar{E}_{i}}(\overline{\mathbf{F}}, \overline{\mathbf{E}}, c)=\widetilde{\epsilon}_{i j}(c) \bar{E}_{j}+O\left(\zeta^{3 / 2}\right),
$$

to leading order. Here, it is important to recognize that the asymptotic constitutive relation (7) for the stress is $O(\zeta)$ while the asymptotic constitutive relation (8) for the electric displacement is of different order, $O\left(\zeta^{1 / 2}\right)$. It is also important to recognize that the permittivity of second order $\widetilde{\tau}$ does not enter in either expression (7) or (8). That is, in this classical limit of small macroscopic deformations and moderate macroscopic electric fields, the overall electromechanical response of dielectric elastomer composites is characterized by three effective tensors: the fourth-order tensor $\widetilde{\mathbf{L}}$ describing their elasticity, the secondorder tensor $\widetilde{\boldsymbol{\epsilon}}$ describing their permittivity, and the fourth-order $\widetilde{\mathbf{M}}$ tensor describing their electrostrictive response.

The object of this paper reduces hence to generating a solution for the effective tensors $\widetilde{\mathbf{L}}, \widetilde{\boldsymbol{\epsilon}}, \widetilde{\mathbf{M}}$ in $(7)$ and (8) directly in terms of the electromechanical properties of the matrix and particles, as characterized by the free energy functions $W^{(1)}$ and $W^{(2)}$, and the microstructure (i.e., the size, the shape, and the spatial distribution of the particles), as characterized by the indicator function $\theta^{(2)}$. To this end, we will make use of a new iterative homogenization theory for elastic dielectric composites recently put forward by LopezPamies (2014). As recalled in more detail further below, this theory provides an exact result for the overall elastic dielectric response of a large class of two-phase particulate composite materials under arbitrarily large deformations and arbitrarily large electric fields. The elastic dielectric behaviors of the underlying matrix and particles are of arbitrary choice, and thus the theory can be utilized to study materials with odd electroelastic coupling (such as piezoelectric materials), as well as with even electroelastic coupling (such as the dielectric elastomers of interest in this work). From an applications standpoint, the theory requires solving a first-order non-linear (Hamilton-Jacobi-type) partial differential equation (pde) in one scalar and two tensorial variables. In this paper, we generate a closed-form solution for such a non-linear pde for the case of materials with even electroelastic coupling in the limit of small deformations and moderate electric fields, thus obtaining a closed-form solution for the sought-after effective tensors $\widetilde{\mathbf{L}}, \widetilde{\boldsymbol{\epsilon}}, \widetilde{\mathbf{M}}$ in (7) and (8).

Here, it is appropriate to record that approximate solutions for the effective tensors $\widetilde{\mathbf{L}}, \widetilde{\boldsymbol{\epsilon}}, \widetilde{\mathbf{M}}$ have been proposed earlier by Li and Rao (2004), Li et al. (2004), and more recently by Siboni and Ponte Castañeda (2013) for the specific case when the particles are mechanically rigid, by making use of classical linear estimates of the Hashin-Shtrikman and self-consistent type. It was in a later contribution that Tian (2007) and Tian et al. (2012) established rigorously via a two-scale convergence argument the homogenization limit of the equations of elastic dielectrics with even electroelastic coupling in the limit of small deformations and moderate electric fields. Remarkably, in spite of the inherent coupling and nonlinearity of the problem, their result indicates that it is possible to write formulae for the effective electromechanical tensors $\widetilde{\mathbf{L}}, \widetilde{\boldsymbol{\epsilon}}, \widetilde{\mathbf{M}}$ in terms of a system of two uncoupled linear pdes. Lefèvre and Lopez-Pamies (2014) have recently solved these pdes analytically and worked out closed-form formulas for the resulting effective tensors $\widetilde{\mathbf{L}}, \widetilde{\boldsymbol{\epsilon}}, \widetilde{\mathbf{M}}$ for the case of isotropic dielectric elastomers filled with an isotropic distribution of polydisperse spherical particles.

The organization of this paper is as follows. For convenience and clarity, the general form of the theory of Lopez-Pamies (2014) together with its salient features are summarized in the next section. Its application to the dielectric elastomer composites with even electromechanical coupling of interest here and the closed-form solution for the effective tensors $\widetilde{\mathbf{L}}, \widetilde{\boldsymbol{\epsilon}}, \widetilde{\mathbf{M}}$ that it generates - equations (37), (38), (39) — are presented in Sections 3 and 4 . Section 4 also includes a discussion of the key theoretical and practical properties of this 
closed-form solution, as well as its specialization to various cases of practical relevance. With the dual aim of gaining physical insight into the proposed solution and shedding light on experiments (Zhang et al., 2002; Huang and Zhang, 2004; Huang et al., 2005; Carpi and Rossi, 2005; McCarthy et al., 2009, Liu et al., 2013), Section 5 confronts the solution to the analytical result of Lefèvre and Lopez-Pamies (2014) and a host of new full-field simulations for the case of dielectric elastomer composites with spherical particles of various elastic dielectric properties (including stiff high-permittivity particles, liquid-like high-permittivity particles, and vacuous pores) that are distributed isotropically. Finally, Appendix A provides the basic details of the full-field simulations presented in Section 5 .

\section{The theory of Lopez-Pamies (2014) for elastic dielectric composites}

By means of a combination of iterative techniques, Lopez-Pamies (2014) has recently generated an exact solution for the variational problem (5) for two-phase elastic dielectric composites with a specific, yet fairly general, class of particulate microstructures. In the present notation, his result for the effective free energy function $\bar{W}=\bar{W}(\overline{\mathbf{F}}, \overline{\mathbf{E}}, c)$ is given implicitly by the first-order nonlinear pde

$$
\mathrm{c} \frac{\partial \bar{W}}{\partial \mathrm{c}}-\bar{W}-\int_{|\boldsymbol{\xi}|=1} \max _{\boldsymbol{\alpha}} \min _{\beta}\left[\boldsymbol{\alpha} \cdot \frac{\partial \bar{W}}{\partial \overline{\mathbf{F}}} \boldsymbol{\xi}+\beta \frac{\partial \bar{W}}{\partial \overline{\mathbf{E}}} \cdot \boldsymbol{\xi}-W^{(1)}(\overline{\mathbf{F}}+\boldsymbol{\alpha} \otimes \boldsymbol{\xi}, \overline{\mathbf{E}}+\beta \boldsymbol{\xi})\right] \nu(\boldsymbol{\xi}) \mathrm{d} \boldsymbol{\xi}=0
$$

subject to the initial condition

$$
\bar{W}(\overline{\mathbf{F}}, \overline{\mathbf{E}}, 1)=W^{(2)}(\overline{\mathbf{F}}, \overline{\mathbf{E}}),
$$

where the integration of the pde (9) is to be carried out from $c=1$ to the desired final value of volume fraction of particles $\mathrm{c}=c$ and the weighting function $\nu(\boldsymbol{\xi})$ in (9) is given in terms of the two-point correlation function $(3)_{2}$ as follows:

- Random microstructures. For the case of random distributions of particles, the function $\nu(\boldsymbol{\xi})$ is given by

$$
\nu(\boldsymbol{\xi})=-\frac{1}{8 \pi^{2}} \int_{\Omega} \frac{p^{(22)}(\mathbf{X})-c^{2}}{(1-c) c} \delta^{\prime \prime}(\boldsymbol{\xi} \cdot \mathbf{X}) \mathrm{d} \mathbf{X},
$$

where $\delta^{\prime \prime}$ denotes the second derivative of the Dirac delta function with respect to its argument. Direct use of relation (11) allows to rewrite the pde (9) more explicitly as

$$
\begin{aligned}
c \frac{\partial \bar{W}}{\partial c}-\bar{W}+\frac{1}{8 \pi^{2}} \int_{|\boldsymbol{\xi}|=1} \int_{\Omega} \max _{\boldsymbol{\alpha}} \min _{\beta}\left[\boldsymbol{\alpha} \cdot \frac{\partial \bar{W}}{\partial \overline{\mathbf{F}}} \boldsymbol{\xi}+\beta \frac{\partial \bar{W}}{\partial \overline{\mathbf{E}}} \cdot \boldsymbol{\xi}-\right. \\
\left.W^{(1)}(\overline{\mathbf{F}}+\boldsymbol{\alpha} \otimes \boldsymbol{\xi}, \overline{\mathbf{E}}+\beta \boldsymbol{\xi})\right] \frac{p^{(22)}(\mathbf{X})-c^{2}}{(1-c) c} \delta^{\prime \prime}(\boldsymbol{\xi} \cdot \mathbf{X}) \mathrm{d} \mathbf{X} \mathrm{d} \boldsymbol{\xi}=0 .
\end{aligned}
$$

- Periodic microstructures. For the case of periodic distributions of particles, the function $\nu(\boldsymbol{\xi})$ is given by

$$
\nu(\boldsymbol{\xi})=\sum_{\mathbf{k} \in \mathcal{R}^{*}-\{\mathbf{0}\}} \frac{\widehat{p}^{(22)}(\mathbf{k})}{(1-c) c} \delta\left(\boldsymbol{\xi}-\frac{\mathbf{k}}{|\mathbf{k}|}\right) \quad \text { with } \quad \widehat{p}^{(22)}(\mathbf{k})=\frac{1}{|Q|} \int_{Q} p^{(22)}(\mathbf{X}) e^{-i \mathbf{X} \cdot \mathbf{k}} \mathrm{d} \mathbf{X}
$$

Here, $\delta(\boldsymbol{\xi}-\mathbf{k} /|\mathbf{k}|)$ denotes the Dirac delta function and $\widehat{p}^{(22)}(\mathbf{k})$ stands for the Fourier transform of the two-point correlation function $p^{(22)}(\mathbf{X})$, while $Q$ denotes the repeating unit cell chosen to describe the microstructure and

$$
\mathcal{R}^{*}=\left\{\mathbf{k}: \mathbf{k}=n_{1} \mathbf{B}_{1}+n_{2} \mathbf{B}_{2}+n_{3} \mathbf{B}_{3}, \quad n_{i} \in \mathbb{Z}\right\}
$$


with

$$
\mathbf{B}_{1}=2 \pi \frac{\mathbf{A}_{2} \wedge \mathbf{A}_{3}}{\mathbf{A}_{1} \cdot\left(\mathbf{A}_{2} \wedge \mathbf{A}_{3}\right)}, \quad \mathbf{B}_{2}=2 \pi \frac{\mathbf{A}_{3} \wedge \mathbf{A}_{1}}{\mathbf{A}_{1} \cdot\left(\mathbf{A}_{2} \wedge \mathbf{A}_{3}\right)}, \quad \mathbf{B}_{3}=2 \pi \frac{\mathbf{A}_{1} \wedge \mathbf{A}_{2}}{\mathbf{A}_{1} \cdot\left(\mathbf{A}_{2} \wedge \mathbf{A}_{3}\right)},
$$

stands for the reciprocal lattice in Fourier space of the periodic lattice in real space

$$
\mathcal{R}=\left\{\mathbf{X}: \mathbf{X}=n_{1} \mathbf{A}_{1}+n_{2} \mathbf{A}_{2}+n_{3} \mathbf{A}_{3}, \quad n_{i} \in \mathbb{Z}\right\}
$$

over which the unit cell $Q$ is repeated. Upon invoking the identity $\widehat{p}^{(22)}(\mathbf{k})=\left|\widehat{\theta}^{(2)}(\mathbf{k})\right|^{2}$ with $\widehat{\theta}^{(2)}(\mathbf{k})=$ $|Q|^{-1} \int_{Q} \theta^{(2)}(\mathbf{X}) e^{-i \mathbf{k} \cdot \mathbf{X}} \mathrm{d} \mathbf{X}$, the pde (9) adopts the more explicit form

$$
c \frac{\partial \bar{W}}{\partial c}-\bar{W}-\sum_{\substack{\mathbf{k} \in \mathcal{R}^{*}-\{\mathbf{0}\} \\ \boldsymbol{\xi}=\mathbf{k} /|\mathbf{k}|}}\left\{\max _{\boldsymbol{\alpha}} \min _{\beta}\left[\boldsymbol{\alpha} \cdot \frac{\partial \bar{W}}{\partial \overline{\mathbf{F}}} \boldsymbol{\xi}+\beta \frac{\partial \bar{W}}{\partial \overline{\mathbf{E}}} \cdot \boldsymbol{\xi}-W^{(1)}(\overline{\mathbf{F}}+\boldsymbol{\alpha} \otimes \boldsymbol{\xi}, \overline{\mathbf{E}}+\beta \boldsymbol{\xi})\right] \frac{\left|\widehat{\theta}^{(2)}(\mathbf{k})\right|^{2}}{(1-c) c}\right\}=0 .
$$

The interested reader is referred to Lopez-Pamies (2014) for the derivation and thorough discussion of the above result. At this stage, nevertheless, it is appropriate to remark that the result (9)-(10) is exact for a specific class of two-phase particulate microstructures and hence it is realizable. Moreover, it is valid for arbitrary ${ }^{1}$ free energy functions $W^{(1)}$ and $W^{(2)}$ and arbitrary one-point $p^{(2)}$ and two-point $p^{(22)}$ correlation functions. Given this generality, the result (9)-(10) can be utilized more broadly as a constitutive theory for two-phase elastic dielectrics with any particulate microstructure: for a given matrix constitutive behavior $W^{(1)}$, given particle constitutive behavior $W^{(2)}$, and given one- and two-point correlation functions $p^{(2)}$ and $p^{(22)}$, the result (9)-(10) provides a constitutive model for the macroscopic response of the elastic dielectric composite of interest.

\section{Application to dielectric elastomer composites}

By virtue of their synthesis and fabrication process into a network of long polymeric chains coiled randomly without a preferred direction, most elastomers are mechanically and dielectrically isotropic. Typically, they are also such that their polarization is proportional to the applied electric field but independent of the applied deformation, much like liquid polymers (see, e.g., Kofod et al., 2003; Wissler and Mazza, 2007; Di Lillo et al., 2011). For our purposes then, based on these observations together with the fact that the ultimate goal of this work is to examine the limit of small deformations and moderate electric fields, it suffices to restrict attention (without loss of generality) to dielectric elastomer composites wherein the matrix material is characterized by a free energy function of the form

$$
W^{(1)}(\mathbf{F}, \mathbf{E})=\frac{\mu}{2}[\mathbf{F} \cdot \mathbf{F}-3]-\mu(J-1)+\frac{\lambda+\mu}{2}(J-1)^{2}-\frac{\varepsilon}{2} \mathbf{F}^{-T} \mathbf{E} \cdot \mathbf{F}^{-T} \mathbf{E} .
$$

Here, $\mathbf{F} \cdot \mathbf{F}=F_{i j} F_{i j}, J=\operatorname{det} \mathbf{F}$, and the parameters $\mu, \lambda, \varepsilon$ stand for the initial Lamé constants and permittivity of the elastomeric material under consideration. On the other hand, the filler particles in dielectric elastomer composites may possibly be anisotropic, both mechanically and dielectrically. That is the case, for instance, for single crystal and textured ceramic particles. No restriction is hence made on the free energy function $W^{(2)}$, other than, again, its even functional dependence on the electric field.

\footnotetext{
${ }^{1}$ As outlined in the Introduction, the theory allows thus to consider elastic dielectric matrix and particles with odd electroelastic coupling, such as piezoelectric materials (Spinelli and Lopez-Pamies, 2014), as well as with even electroelastic coupling, such as the dielectric elastomers of interest in this work.
} 
Now, for the matrix free energy function (18), the maximizing vector $\boldsymbol{\alpha}$ and minimizing scalar $\beta$ in (9) can be determined in closed form. They read as

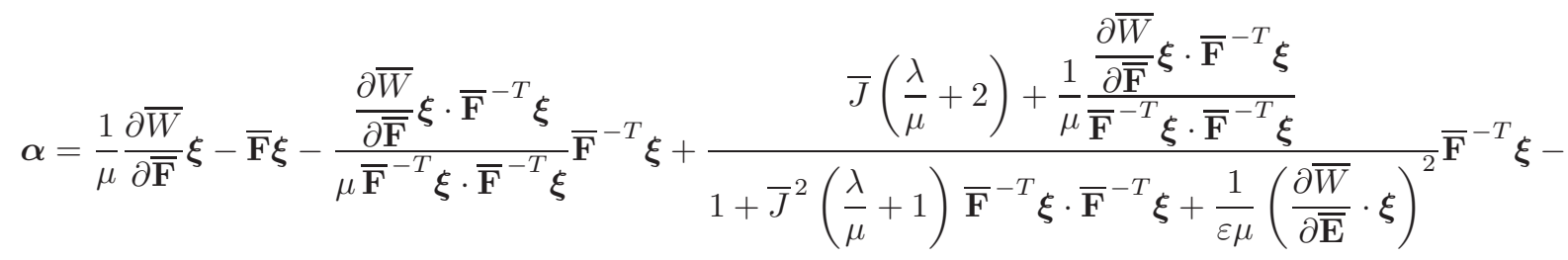

$$
\begin{aligned}
& \frac{\left(\frac{\partial \bar{W}}{\partial \overline{\mathbf{E}}} \cdot \boldsymbol{\xi}\right)\left(\overline{\mathbf{F}}^{-T} \overline{\mathbf{E}} \cdot \overline{\mathbf{F}}^{-T} \boldsymbol{\xi}\right)}{\mu \overline{\mathbf{F}}^{-T} \boldsymbol{\xi} \cdot \overline{\mathbf{F}}^{-T} \boldsymbol{\xi}} \overline{\mathbf{F}}^{-T} \boldsymbol{\xi}+\frac{1}{\mu}\left(\frac{\partial \bar{W}}{\partial \overline{\mathbf{E}}} \cdot \boldsymbol{\xi}\right) \overline{\mathbf{F}}^{-T} \overline{\mathbf{E}}
\end{aligned}
$$

and

$$
\beta=\boldsymbol{\alpha} \cdot \overline{\mathbf{F}}^{-T} \overline{\mathbf{E}}-\frac{\left(1+\boldsymbol{\alpha} \cdot \overline{\mathbf{F}}^{-T} \boldsymbol{\xi}\right)^{2}}{\varepsilon \overline{\mathbf{F}}^{-T} \boldsymbol{\xi} \cdot \overline{\mathbf{F}}^{-T} \boldsymbol{\xi}}\left[\frac{\partial \bar{W}}{\partial \overline{\mathbf{E}}} \cdot \boldsymbol{\xi}+\frac{\varepsilon \overline{\mathbf{F}}^{-T} \overline{\mathbf{E}} \cdot \overline{\mathbf{F}}^{-T} \boldsymbol{\xi}}{1+\boldsymbol{\alpha} \cdot \overline{\mathbf{F}}^{-T} \boldsymbol{\xi}}\right],
$$

where $\bar{J} \doteq \operatorname{det} \overline{\mathbf{F}}$ has been introduced to ease notation. Direct use of relations (19)-(20) in expression (9), together with some lengthy but straightforward calculations, allows to write the solution for the effective free energy function in the more explicit form

$$
\bar{W}(\overline{\mathbf{F}}, \overline{\mathbf{E}}, c)=\bar{U}(\overline{\mathbf{F}}, \overline{\mathbf{E}}, c)+\frac{\mu}{2}[\overline{\mathbf{F}} \cdot \overline{\mathbf{F}}-3]-\mu(\bar{J}-1)+\frac{\lambda+\mu}{2}(\bar{J}-1)^{2}-\frac{\varepsilon}{2} \overline{\mathbf{F}}^{-T} \overline{\mathbf{E}} \cdot \overline{\mathbf{F}}^{-T} \overline{\mathbf{E}},
$$

where the function $\bar{U}$ is defined implicitly as the solution to the pde

$$
\begin{aligned}
& 2 \mu \mathrm{c} \frac{\partial \bar{U}}{\partial \mathrm{c}}-2 \mu \bar{U}-\int_{|\boldsymbol{\xi}|=1}\left\{\frac{\partial \bar{U}}{\partial \overline{\mathbf{F}}} \boldsymbol{\xi} \cdot \frac{\partial \bar{U}}{\partial \overline{\mathbf{F}}} \boldsymbol{\xi}-\left[1-\frac{\varepsilon \mu}{\Upsilon}\right] \frac{\left(\frac{\partial \bar{U}}{\partial \overline{\mathbf{F}}} \boldsymbol{\xi} \cdot \overline{\mathbf{F}}^{-T} \boldsymbol{\xi}\right)^{2}}{\overline{\mathbf{F}}^{-T} \boldsymbol{\xi} \cdot \overline{\mathbf{F}}^{-T} \boldsymbol{\xi}}-\left[\frac{\mu}{\varepsilon}+\left(\overline{\mathbf{F}}^{-T} \overline{\mathbf{E}} \cdot \overline{\mathbf{F}}^{-T} \boldsymbol{\xi}\right)^{2}-\right.\right. \\
& \left.\left(\overline{\mathbf{F}}^{-T} \overline{\mathbf{E}} \cdot \overline{\mathbf{F}}^{-T} \overline{\mathbf{E}}\right)\left(\overline{\mathbf{F}}^{-T} \boldsymbol{\xi} \cdot \overline{\mathbf{F}}^{-T} \boldsymbol{\xi}\right)-\frac{\mu}{\varepsilon \Upsilon}\left(\frac{\partial \bar{U}}{\partial \overline{\mathbf{E}}} \cdot \boldsymbol{\xi}-2 \varepsilon \overline{\mathbf{F}}^{-T} \overline{\mathbf{E}} \cdot \overline{\mathbf{F}}^{-T} \boldsymbol{\xi}\right)^{2}\right] \frac{\left(\frac{\partial \bar{U}}{\partial \overline{\mathbf{E}}} \cdot \boldsymbol{\xi}\right)^{2}}{\overline{\mathbf{F}}^{-T} \boldsymbol{\xi} \cdot \overline{\mathbf{F}}^{-T} \boldsymbol{\xi}}+2\left[\frac{\partial \bar{U}}{\partial \overline{\mathbf{F}}} \boldsymbol{\xi} \cdot \overline{\mathbf{F}}^{-T} \overline{\mathbf{E}}+\right. \\
& \left.\left.\left[\frac{\mu}{\Upsilon}\left(2 \varepsilon \overline{\mathbf{F}}^{-T} \overline{\mathbf{E}} \cdot \overline{\mathbf{F}}^{-T} \boldsymbol{\xi}-\frac{\partial \bar{U}}{\partial \overline{\mathbf{E}}} \cdot \boldsymbol{\xi}\right)-\overline{\mathbf{F}}^{-T} \overline{\mathbf{E}} \cdot \overline{\mathbf{F}}^{-T} \boldsymbol{\xi}\right] \frac{\left(\frac{\partial \bar{U}}{\partial \overline{\mathbf{F}}} \boldsymbol{\xi} \cdot \overline{\mathbf{F}}^{-T} \boldsymbol{\xi}\right)}{\overline{\mathbf{F}}^{-T} \boldsymbol{\xi} \cdot \overline{\mathbf{F}}^{-T} \boldsymbol{\xi}}\right]\left(\frac{\partial \bar{U}}{\partial \overline{\mathbf{E}}} \cdot \boldsymbol{\xi}\right)\right\} \nu(\boldsymbol{\xi}) \mathrm{d} \boldsymbol{\xi}=0,
\end{aligned}
$$

with

$$
\Upsilon=\varepsilon \mu+\left(\frac{\partial \bar{U}}{\partial \overline{\mathbf{E}}} \cdot \boldsymbol{\xi}-\varepsilon \overline{\mathbf{F}}^{-T} \overline{\mathbf{E}} \cdot \overline{\mathbf{F}}^{-T} \boldsymbol{\xi}\right)^{2}+\varepsilon \bar{J}^{2}(\lambda+\mu) \overline{\mathbf{F}}^{-T} \boldsymbol{\xi} \cdot \overline{\mathbf{F}}^{-T} \boldsymbol{\xi},
$$

subject to the initial condition

$$
\bar{U}(\overline{\mathbf{F}}, \overline{\mathbf{E}}, 1)=W^{(2)}(\overline{\mathbf{F}}, \overline{\mathbf{E}})-\frac{\mu}{2}[\overline{\mathbf{F}} \cdot \overline{\mathbf{F}}-3]+\mu(\bar{J}-1)-\frac{\lambda+\mu}{2}(\bar{J}-1)^{2}+\frac{\varepsilon}{2} \overline{\mathbf{F}}^{-T} \overline{\mathbf{E}} \cdot \overline{\mathbf{F}}^{-T} \overline{\mathbf{E}} .
$$

The computation of the effective free energy function $\bar{W}$ amounts thus to solving the initial-value problem (22)-(24) for $\bar{U}$. In view of the quadratic nonlinearity of the pde (22), this initial-value problem might admit a closed-form solution, at least for some choices of particle constitutive behaviors and microstructures (see, e.g., Lopez-Pamies et al., 2013a). The analytical solvability and properties of the pde (22) in its general form, however, is a substantial task deferred for presentation elsewhere. In the sequel, we restrict our attention to its asymptotic behavior in the limit of small deformations and moderate electric fields. 


\section{Asymptotic solution in the limit of small deformations and moderate electric fields}

The result (21) with (22)-(24) for the effective free energy function $\bar{W}$ of dielectric elastomer composites is valid for arbitrarily large macroscopic deformation gradients $\overline{\mathbf{F}}$ and arbitrarily large macroscopic electric fields $\overline{\mathbf{E}}$. In this section, we examine its asymptotic behavior in the classical limit of small deformations and moderate electric fields, as described in Section 1.1.

Before proceeding with the pertinent details, it proves convenient to introduce the following quantities:

$$
\begin{aligned}
& \mathbf{L}^{(1)} \doteq \frac{\partial^{2} W^{(1)}}{\partial \mathbf{F}^{2}}(\mathbf{I}, \mathbf{0})=2 \mu \mathcal{K}+(3 \lambda+2 \mu) \mathcal{J}, \quad \boldsymbol{\epsilon}^{(1)} \doteq-\frac{\partial^{2} W^{(1)}}{\partial \mathbf{E}^{2}}(\mathbf{I}, \mathbf{0})=\varepsilon \mathbf{I}, \quad \mathbf{M}^{(1)} \doteq \frac{1}{2} \frac{\partial^{3} W^{(1)}}{\partial \mathbf{F} \partial \mathbf{E}^{2}}(\mathbf{I}, \mathbf{0})=\varepsilon \mathcal{I}, \\
& \mathbf{L}^{(2)} \doteq \frac{\partial^{2} W^{(2)}}{\partial \mathbf{F}^{2}}(\mathbf{I}, \mathbf{0}), \quad \boldsymbol{\epsilon}^{(2)} \doteq-\frac{\partial^{2} W^{(2)}}{\partial \mathbf{E}^{2}}(\mathbf{I}, \mathbf{0}), \quad \mathbf{M}^{(2)} \doteq \frac{1}{2} \frac{\partial^{3} W^{(2)}}{\partial \mathbf{F} \partial \mathbf{E}^{2}}(\mathbf{I}, \mathbf{0}), \\
& \Delta \mathbf{L}=\mathbf{L}^{(2)}-\mathbf{L}^{(1)}, \quad \Delta \boldsymbol{\epsilon}=\boldsymbol{\epsilon}^{(2)}-\boldsymbol{\epsilon}^{(1)}, \quad \Delta \mathbf{M}=\mathbf{M}^{(2)}-\mathbf{M}^{(1)},
\end{aligned}
$$

where the tensors $\mathcal{K}, \mathcal{J}$ are given in component form by

$$
\mathcal{K}_{i j k l}=\frac{1}{2}\left[\delta_{i k} \delta_{j l}+\delta_{i l} \delta_{j k}-\frac{2}{3} \delta_{i j} \delta_{k l}\right], \quad \mathcal{J}_{i j k l}=\frac{1}{3} \delta_{i j} \delta_{k l}
$$

$\mathcal{I}$ stands for the identity in the space of fourth-order tensors with major and minor symmetries, $\mathcal{I}_{i j k l}=$ $1 / 2\left(\delta_{i k} \delta_{j l}+\delta_{i l} \delta_{j k}\right)$, and $\delta_{i j}$ denotes the Kronecker delta. For later reference, we remark that $\mathcal{K}$ and $\mathcal{J}$ are orthogonal projection tensors with the properties $\mathcal{K} \mathcal{J}=\mathcal{J K}=\mathbf{0}, \mathcal{K} \mathcal{K}=\mathcal{K}, \mathcal{J} \mathcal{J}=\mathcal{J}, \mathcal{K}+\mathcal{J}=\mathcal{I}$. The tensors $\mathbf{L}^{(r)}, \boldsymbol{\epsilon}^{(r)}, \mathbf{M}^{(r)}$ introduced in (25)-(26) correspond physically to the modulus of elasticity, the permittivity, and the electrostrictive tensor of the matrix $(r=1)$ and particles $(r=2)$. Due to the energy character and objectivity of $W^{(r)}$, they possess the symmetries $L_{i j k l}^{(r)}=L_{k l i j}^{(r)}=L_{j i k l}^{(r)}=L_{i j l k}^{(r)}, \epsilon_{i j}^{(r)}=\epsilon_{j i}^{(r)}$, $M_{i j k l}^{(r)}=M_{j i k l}^{(r)}=M_{i j l k}^{(r)}$.

We begin the asymptotic analysis by recognizing that the series expansion of the pde (22) and initial condition (24) about $\overline{\mathbf{H}}=\mathbf{0}$ (recall that $\overline{\mathbf{H}}=\overline{\mathbf{F}}-\mathbf{I}$ ) and $\overline{\mathbf{E}}=\mathbf{0}$ leads to a hierarchical system of differential equations associated with powers $\overline{\mathbf{H}}^{m} \overline{\mathbf{E}}^{2 n} m, n=0,1,2, \ldots \infty$. In the limit as $\zeta \rightarrow 0$, when the deformation measure $\overline{\mathbf{H}}$ is taken to be $O(\zeta)$ and the electric field $\overline{\mathbf{E}}$ is taken to be $O\left(\zeta^{1 / 2}\right)$, it follows from the equations associated with the powers $\overline{\mathbf{H}}^{2}, \overline{\mathbf{E}}^{2}, \overline{\mathbf{H}} \overline{\mathbf{E}}^{2}$ that the effective free energy function (21) is of the form (6), where

the effective tensors $\widetilde{\mathbf{L}}, \widetilde{\boldsymbol{\epsilon}}, \widetilde{\mathbf{M}}$ are defined by the system of coupled nonlinear ordinary differential equations (odes)

$$
\begin{aligned}
& \mathrm{c} \frac{\mathrm{d} \widetilde{L}_{i j k l}}{\mathrm{dc}}-\Delta \widetilde{L}_{i j k l}-\Delta \widetilde{L}_{i j p q} P_{p q m n}^{L} \Delta \widetilde{L}_{m n k l}=0, \\
& \mathrm{c} \frac{\mathrm{d} \widetilde{\epsilon}_{i j}}{\mathrm{dc}}-\Delta \widetilde{\epsilon}_{i j}-\Delta \widetilde{\epsilon}_{i p} P_{p q}^{\epsilon} \Delta \widetilde{\epsilon}_{q j}=0, \\
& \mathrm{c} \frac{\mathrm{d} \widetilde{M}_{i j k l}}{\mathrm{dc}}-\Delta \widetilde{M}_{i j k l}-\Delta \widetilde{L}_{i j p q} P_{p q m n}^{L} \Delta \widetilde{M}_{m n k l}-\Delta \widetilde{M}_{i j k p} P_{p r}^{\epsilon} \Delta \widetilde{\epsilon}_{r l}-\Delta \widetilde{M}_{i j l p} P_{p r}^{\epsilon} \Delta \widetilde{\epsilon}_{r k}+Q_{i j k l}^{M}=0,
\end{aligned}
$$

subject to the initial conditions

$$
\widetilde{\mathbf{L}}(1)=\mathbf{L}^{(2)}, \quad \widetilde{\boldsymbol{\epsilon}}(1)=\boldsymbol{\epsilon}^{(2)}, \quad \widetilde{\mathbf{M}}(1)=\mathbf{M}^{(2)} .
$$

In the above expressions, we have made use of the notation $\Delta \widetilde{\mathbf{L}}=\widetilde{\mathbf{L}}-\mathbf{L}^{(1)}, \Delta \widetilde{\boldsymbol{\epsilon}}=\widetilde{\boldsymbol{\epsilon}}-\boldsymbol{\epsilon}^{(1)}, \Delta \widetilde{\mathbf{M}}=\widetilde{\mathbf{M}}-\mathbf{M}^{(1)}$, 
and $\mathbf{P}^{L}, \mathbf{P}^{\epsilon}, \mathbf{Q}^{M}$ are microstructural tensors given in component form by

$$
\begin{aligned}
P_{i j k l}^{L}= & \left.\frac{1}{\mu} \delta_{i k}\left\langle\xi_{j} \xi_{l}\right\rangle\right|_{(i j),(k l)}-\frac{\mu+\lambda}{\mu(2 \mu+\lambda)}\left\langle\xi_{i} \xi_{j} \xi_{k} \xi_{l}\right\rangle, \\
P_{i j}^{\epsilon}= & \frac{1}{\varepsilon}\left\langle\xi_{i} \xi_{j}\right\rangle, \\
Q_{i j k l}^{M}= & \frac{1}{\varepsilon(2 \mu+\lambda)} \Delta \widetilde{L}_{i j p q} \Delta \widetilde{\epsilon}_{m k} \Delta \widetilde{\epsilon}_{n l}\left\langle\xi_{p} \xi_{q} \xi_{m} \xi_{n}\right\rangle+\frac{1}{\varepsilon} \Delta \widetilde{\epsilon}_{p k} \Delta \widetilde{\epsilon}_{q l}\left\langle\xi_{i} \xi_{j} \xi_{p} \xi_{q}\right\rangle- \\
& \left.\frac{\lambda}{\mu(2 \mu+\lambda)} \Delta \widetilde{L}_{i j p q} \Delta \widetilde{\epsilon}_{m l}\left\langle\xi_{p} \xi_{q} \xi_{m} \xi_{k}\right\rangle\right|_{(k l)}+\left.\frac{1}{\mu} \Delta \widetilde{L}_{i j k q} \Delta \widetilde{\epsilon}_{p l}\left\langle\xi_{p} \xi_{q}\right\rangle\right|_{(k l)},
\end{aligned}
$$

where the bracketed subscripts imply symmetrization and the symbol

$$
\langle\cdot\rangle \doteq \int_{|\boldsymbol{\xi}|=1} \cdot \nu(\boldsymbol{\xi}) \mathrm{d} \boldsymbol{\xi}
$$

has been introduced for further notational simplicity; recall from Section 2 that the weighting function $\nu(\boldsymbol{\xi})$ is given directly in terms of the two-point correlation function $p^{(22)}$ by expression (11) for random microstructures and by (13) for periodic ones.

From a computational point of view, it is useful to recognize that the odes (29) and (30), with initial conditions $(32)_{1}$ and $(32)_{2}$, for the effective modulus of elasticity $\widetilde{\mathbf{L}}$ and effective permittivity $\widetilde{\boldsymbol{\epsilon}}$ are nonlinear Riccati equations uncoupled from each other and from the ode (31) for the effective electrostrictive tensor $\widetilde{\mathbf{M}}$. In spite of their quadratic nonlinearity, they can be solved in closed form. Their solutions read simply as

$$
\widetilde{L}_{i j k l}=\mu\left(\delta_{i k} \delta_{j l}+\delta_{i l} \delta_{j k}\right)+\lambda \delta_{i j} \delta_{k l}+c\left\{\left[(1-c) \mathbf{P}^{L}+\Delta \mathbf{L}^{-1}\right]^{-1}\right\}_{i j k l}
$$

and

$$
\widetilde{\epsilon}_{i j}=\varepsilon \delta_{i j}+c\left\{\left[(1-c) \mathbf{P}^{\epsilon}+\Delta \boldsymbol{\epsilon}^{-1}\right]^{-1}\right\}_{i j} .
$$

Having determined the results (37) and (38), the linear ode (31), which does depend on $\widetilde{\mathbf{L}}$ and $\widetilde{\boldsymbol{\epsilon}}$, can also be solved in closed form to render

$$
\begin{aligned}
\widetilde{M}_{i j k l}= & \frac{\varepsilon}{2}\left(\delta_{i k} \delta_{j l}+\delta_{i l} \delta_{j k}\right)+\frac{1}{c^{2}} \Delta \widetilde{L}_{i j m n} \Delta L_{m n p q}^{-1} \Delta M_{p q r s} \Delta \epsilon_{r u}^{-1} \Delta \widetilde{\epsilon}_{u k} \Delta \epsilon_{s v}^{-1} \Delta \widetilde{\epsilon}_{v l}+\frac{1-c}{2 c \varepsilon} \Delta \widetilde{\epsilon}_{k r} \Delta \widetilde{\epsilon}_{l s}\left\langle\xi_{i} \xi_{j} \xi_{r} \xi_{s}\right\rangle- \\
& \frac{c^{2}-1}{2 c^{2} \varepsilon(2 \mu+\lambda)} \Delta \widetilde{L}_{i j p q} \Delta \widetilde{\epsilon}_{k r} \Delta \widetilde{\epsilon}_{l s}\left\langle\xi_{p} \xi_{q} \xi_{r} \xi_{s}\right\rangle+\frac{1-c}{2 c^{2} \varepsilon} \Delta \widetilde{L}_{i j p q} \Delta L_{p q m n}^{-1} \Delta \widetilde{\epsilon}_{k r} \Delta \widetilde{\epsilon}_{l s}\left\langle\xi_{m} \xi_{n} \xi_{r} \xi_{s}\right\rangle- \\
& \left.\frac{\lambda(1-c)}{2 c^{2} \mu(2 \mu+\lambda)} \Delta \widetilde{L}_{i j p q}\left[c \delta_{m k}+\Delta \epsilon_{m r}^{-1} \Delta \widetilde{\epsilon}_{k r}\right] \Delta \widetilde{\epsilon}_{l s}\left\langle\xi_{p} \xi_{q} \xi_{m} \xi_{s}\right\rangle\right|_{(k l)}+ \\
& \left.\frac{1-c}{2 c^{2} \mu} \Delta \widetilde{L}_{i j p q}\left[c \delta_{p k}+\Delta \epsilon_{p r}^{-1} \Delta \widetilde{\epsilon}_{k r}\right] \Delta \widetilde{\epsilon}_{l s}\left\langle\xi_{s} \xi_{q}\right\rangle\right|_{(k l)} .
\end{aligned}
$$

The exact closed-form solutions (37), (38), (39) constitute the main result of this paper. They characterize the overall electromechanical response of dielectric elastomer composites with a large class of random and periodic particulate microstructures in the limit of small deformations and moderate electric fields. The following theoretical and practical remarks are in order:

i. Elastic dielectric behaviors of the matrix and particles. The solutions (37), (38), (39) are valid for any choice of Lamé constants $\mu, \lambda$ and permittivity $\varepsilon$ describing the isotropic elastic dielectric behavior of the elastomeric matrix, as well as for any choice of modulus of elasticity $\mathbf{L}^{(2)}$, permittivity $\boldsymbol{\epsilon}^{(2)}$, and electrostrictive tensor $\mathbf{M}^{(2)}$ describing the (possibly anisotropic) elastic dielectric behavior of the underlying particles. 
For the practically relevant case when the particles are isotropic ideal elastic dielectrics with

$$
\mathbf{L}^{(2)}=2 \mu_{p} \mathcal{K}+\left(3 \lambda_{p}+2 \mu_{p}\right) \mathcal{J}, \quad \boldsymbol{\epsilon}^{(2)}=\varepsilon_{p} \mathbf{I}, \quad \mathbf{M}^{(2)}=\varepsilon_{p} \mathcal{I},
$$

where $\mu_{p}, \lambda_{p}, \varepsilon_{p}$ denote the Lamé constants and permittivity of the particles, the solutions (37), (38), (39) specialize to

$$
\begin{aligned}
& \widetilde{L}_{i j k l}= \mu\left(\delta_{i k} \delta_{j l}+\delta_{i l} \delta_{j k}\right)+\lambda \delta_{i j} \delta_{k l}+c\left\{\left[(1-c) \mathbf{P}^{L}+\frac{1}{2 \Delta \mu} \mathcal{K}+\frac{1}{3 \Delta \lambda+2 \Delta \mu} \mathcal{J}\right]^{-1}\right\}_{i j k l} \\
& \widetilde{\epsilon}_{i j}=\varepsilon \delta_{i j}+c\left\{\left[(1-c) \mathbf{P}^{\epsilon}+\frac{1}{\Delta \varepsilon} \mathbf{I}\right]^{-1}\right\}_{i j}, \\
& \widetilde{M}_{i j k l}= \varepsilon \mathcal{I}_{i j k l}+\frac{1}{2 c^{2} \Delta \mu \Delta \varepsilon} \Delta \widetilde{L}_{i j p q} \Delta \widetilde{\epsilon}_{k p} \Delta \widetilde{\epsilon}_{l q}-\frac{1}{2 c^{2} \Delta \varepsilon}\left[\frac{\Delta \lambda}{\Delta \mu(3 \Delta \lambda+2 \Delta \mu)}\right] \Delta \widetilde{L}_{i j m m} \Delta \widetilde{\epsilon}_{k r} \Delta \widetilde{\epsilon}_{r l}- \\
& \frac{1}{2 c^{2}}\left[\frac{c^{2}-1}{\varepsilon(2 \mu+\lambda)}-\frac{1-c}{2 \varepsilon \Delta \mu}+\frac{\lambda(1-c)}{\mu(2 \mu+\lambda) \Delta \varepsilon}\right] \Delta \widetilde{L}_{i j p q} \Delta \widetilde{\epsilon}_{k r} \Delta \widetilde{\epsilon}_{l s}\left\langle\xi_{p} \xi_{q} \xi_{r} \xi_{s}\right\rangle- \\
& \frac{1-c}{4 c^{2} \varepsilon}\left[\frac{\Delta \lambda}{\Delta \mu(3 \Delta \lambda+2 \Delta \mu)}\right] \Delta \widetilde{L}_{i j m m} \Delta \widetilde{\epsilon}_{k r} \Delta \widetilde{\epsilon}_{l s}\left\langle\xi_{r} \xi_{s}\right\rangle+\frac{1-c}{2 c \varepsilon} \Delta \widetilde{\epsilon}_{k r} \Delta \widetilde{\epsilon}_{l s}\left\langle\xi_{i} \xi_{j} \xi_{r} \xi_{s}\right\rangle+ \\
&\left.\frac{1-c}{2 c^{2} \mu \Delta \varepsilon} \Delta \widetilde{L}_{i j p q} \Delta \widetilde{\epsilon}_{k r} \Delta \widetilde{\epsilon}_{l p}\left\langle\xi_{r} \xi_{q}\right\rangle\right|_{(k l)}-\left.\frac{\lambda(1-c)}{2 c \mu(2 \mu+\lambda)} \Delta \widetilde{L}_{i j p q} \Delta \widetilde{\epsilon}_{l s}\left\langle\xi_{p} \xi_{q} \xi_{k} \xi_{s}\right\rangle\right|_{(k l)}+ \\
&\left.\frac{1-c}{2 c \mu} \Delta \widetilde{L}_{i j k q} \Delta \widetilde{\epsilon}_{l s}\left\langle\xi_{s} \xi_{q}\right\rangle\right|_{(k l)},
\end{aligned}
$$

where use has been made of the notation $\Delta \mu=\mu_{p}-\mu, \Delta \lambda=\lambda_{p}-\lambda, \Delta \varepsilon=\varepsilon_{p}-\varepsilon$, and the fact that $\mathcal{K}$ and $\mathcal{J}$ are orthogonal projection tensors.

ii. Geometry and spatial distribution of the particles. The solutions (37), (38), (39) are also valid for any choice of the one- and two-point correlation functions $p^{(2)}=c$ and $p^{(22)}$ describing the microstructure. In practice, both of these quantities are generally measurable and often times readily known from the outset. In particular, the information on $p^{(22)}$ enters the solutions (37), (38), (39) through the microstructural tensors $\langle\boldsymbol{\xi} \otimes \boldsymbol{\xi}\rangle$ and $\langle\boldsymbol{\xi} \otimes \boldsymbol{\xi} \otimes \boldsymbol{\xi} \otimes \boldsymbol{\xi}\rangle$. For demonstration purposes and later use, we spell out next the specialization of these tensors to two basic cases: $(i)$ a random distribution of aligned ellipsoidal particles and (ii) a periodic distribution of ellipsoidal particles. Figure 1 depicts schematics of these two microstructures with the various quantities of interest indicated.

(a)

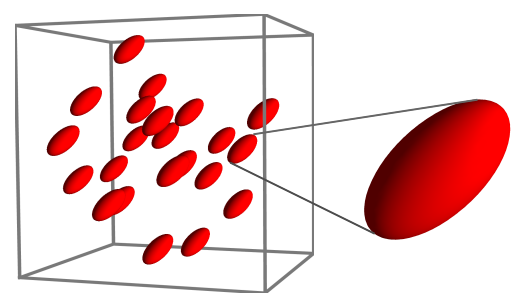

(b)

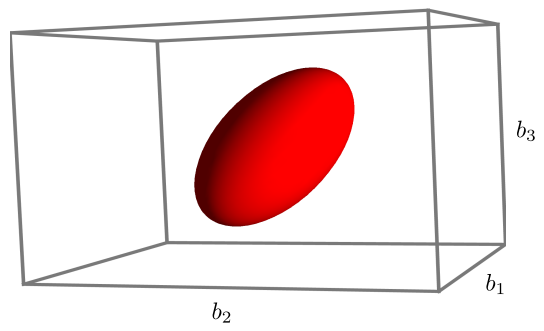

Figure 1: (a) Schematic of a random distribution of aligned ellipsoidal particles. (b) Rectangular prismatic unit cell, with principal axes $\mathbf{u}_{1}, \mathbf{u}_{2}, \mathbf{u}_{3}$ and sides $b_{1}, b_{2}, b_{3}$, describing a periodic cuboidal distribution of ellipsoidal particles.

For microstructures comprised of aligned ellipsoidal particles whose centers are distributed randomly with the so-called "ellipsoidal" symmetry introduced by Willis (1977), the weighting function (11) can 
be determined explicitly allowing to write

$$
\begin{aligned}
& \left\langle\xi_{i} \xi_{j} \xi_{k} \xi_{l}\right\rangle=\frac{\operatorname{det} \mathbf{Z}}{4 \pi} \int_{|\boldsymbol{\xi}|=1} \frac{\xi_{i} \xi_{j} \xi_{k} \xi_{l}}{|\mathbf{Z} \boldsymbol{\xi}|^{3}} \mathrm{~d} \boldsymbol{\xi} \\
& \left\langle\xi_{i} \xi_{j}\right\rangle=\frac{\operatorname{det} \mathbf{Z}}{4 \pi} \int_{|\boldsymbol{\xi}|=1} \frac{\xi_{i} \xi_{j}}{|\mathbf{Z} \boldsymbol{\xi}|^{3}} \mathrm{~d} \boldsymbol{\xi} .
\end{aligned}
$$

Here, the second-order tensor $\mathbf{Z}$ serves to characterize both the shape and alignment of the particles, as well as the "ellipsoidal" spatial distribution of their centers. In particular, the domain occupied by a particle centered at $\mathbf{X}_{c}$ is described by the equation $\Omega_{c}^{(2)}=\left\{\mathbf{X}:\left(\mathbf{X}-\mathbf{X}_{c}\right) \cdot \mathbf{Z}^{-T} \mathbf{Z}^{-1}\left(\mathbf{X}-\mathbf{X}_{c}\right) \leq 1\right\}$. Expressions (44) contain three limiting cases worth remarking. Setting $\mathbf{Z}=\mathbf{I}$ corresponds to an isotropic distribution of spherical particles. Taking the limit of one of the axes of the ellipsoidal particles to be infinitely long corresponds to a distribution of aligned cylindrical fibers with elliptical cross section. Taking the limit of two of the axes of the ellipsoidal particles to be infinitely long corresponds, in turn, to a random distribution of aligned layers (see, e.g., Section 2.13 in LopezPamies, 2006 for technical details on these limits).

For microstructures comprised of a periodic distribution of ellipsoidal particles where the repeating unit cell is a rectangular prism of sides $b_{1}, b_{2}, b_{3}$ containing a single particle located at its center, it is not difficult to compute the Fourier transform $(13)_{2}$ of its two-point correlation function and reciprocal lattice (14)-(15) in order to deduce that

$$
\begin{aligned}
& \left\langle\xi_{i} \xi_{j} \xi_{k} \xi_{l}\right\rangle=\frac{c}{1-c} \sum_{\substack{\mathrm{p}_{1}=-\infty \\
-\left\{\mathrm{p}_{1}=\mathrm{p}_{2}=\mathrm{p}_{3}=0\right\}}}^{\infty} \sum_{\substack{\mathrm{p}_{3}=-\infty \\
-}}^{\infty} \frac{9(\sin |\mathbf{Z} \boldsymbol{\xi}|-|\mathbf{Z} \boldsymbol{\xi}| \cos |\mathbf{Z} \boldsymbol{\xi}|)^{2} \xi_{i} \xi_{j} \xi_{k} \xi_{l}}{|\boldsymbol{\xi}|^{4}|\mathbf{Z} \boldsymbol{\xi}|^{6}}, \\
& \left\langle\xi_{i} \xi_{j}\right\rangle=\frac{c}{1-c} \sum_{\substack{\mathrm{p}_{1}=-\infty \\
-\left\{\mathrm{p}_{1}=\mathrm{p}_{2}=\mathrm{p}_{3}=0\right\}}}^{\infty} \sum_{\mathrm{p}_{3}=-\infty}^{\infty} \frac{9(\sin |\mathbf{Z} \boldsymbol{\xi}|-|\mathbf{Z} \boldsymbol{\xi}| \cos |\mathbf{Z} \boldsymbol{\xi}|)^{2} \xi_{i} \xi_{j}}{|\boldsymbol{\xi}|^{2}|\mathbf{Z} \boldsymbol{\xi}|^{6}} .
\end{aligned}
$$

In these expressions, $\mathbf{Z}$ describes, as in the foregoing, the shape and alignment of the particles, $c=$ $4 \pi \operatorname{det} \mathbf{Z} / 3 b_{1} b_{2} b_{3}$, and

$$
\boldsymbol{\xi}=\frac{2 \pi}{b_{1}} \mathrm{p}_{1} \mathbf{u}_{1}+\frac{2 \pi}{b_{2}} \mathrm{p}_{2} \mathbf{u}_{2}+\frac{2 \pi}{b_{3}} \mathrm{p}_{3} \mathbf{u}_{3}
$$

in terms of the summation integers $\mathrm{p}_{1}, \mathrm{p}_{2}, \mathrm{p}_{3}$, where the mutually orthogonal unit vectors $\mathbf{u}_{1}, \mathbf{u}_{2}, \mathbf{u}_{3}$ stand for the principal axes of the unit cell, as depicted in Fig. 1(b). Setting $\mathbf{Z}=\mathbf{I}$ in (45) corresponds to a periodic distribution of spherical particles. The limiting cases of a periodic distribution of aligned cylindrical fibers with elliptical cross section and a periodic distribution of aligned layers are also contained in expressions (45).

iii. Connection with the Hashin-Shtrikman variational principles in elastostatics and electrostatics. By construction, the underlying microstructure associated with the solutions (37), (38), (39) corresponds to a distribution of disconnected particles that interact in such a manner that their deformation gradient and electric field - irrespectively of the value of the volume fraction of particles $c$ - are uniform and the same in each particle (see Appendix B in Lopez-Pamies, 2014).

An interesting implication of such a special type of intra-particle fields is that the solutions (37) and (38) for the effective modulus of elasticity $\widetilde{\mathbf{L}}$ and effective permittivity $\widetilde{\boldsymbol{\epsilon}}$ agree identically with the variational approximations obtained from the Hashin-Shtrikman variational principles in elastostatics (Hashin and Shtrikman, 1962a) and electrostatics (Hashin and Shtrikman, 1962b) when choosing the reference medium to coincide with the matrix material and the trial polarization field to be constant per phase (so that the fields within the particles are also constant). A corollary of this agreement is that the solutions (37) and (38) coincide identically with one of the Hashin-Shtrikman bounds when 
the elastic and dielectric properties of the matrix and particles are well ordered: the result (37) agrees with the upper (lower) bound in elastostatics when $\mathbf{L}^{(2)}<\mathbf{L}^{(1)}\left(\mathbf{L}^{(2)}>\mathbf{L}^{(1)}\right)$ in the sense of quadratic forms, while the result (38) agrees with the upper (lower) bound in electrostatics when $\boldsymbol{\epsilon}^{(2)}<\boldsymbol{\epsilon}^{(1)}$ $\left(\epsilon^{(2)}>\epsilon^{(1)}\right)$ also in the sense of quadratic forms. In view of these connections, it would be interesting to explore in future studies whether the solution (39) for the effective electrostrictive tensor $\widetilde{\mathbf{M}}$ possesses similar extremal properties.

iv. Connection with the classical results for dilute suspensions of ellipsoidal particles in elastostatics and electrostatics. A further implication of the uniformity of the intra-particle fields is that the solutions (37) and (38) agree with the classical results for the effective modulus of elasticity $\widetilde{\mathbf{L}}$ (Eshelby, 1957) and effective permittivity $\widetilde{\boldsymbol{\epsilon}}$ (see, e.g., Bergman, 1978 and references therein) of a dilute suspension of aligned ellipsoidal particles. By contrast, through a comparison with an exact solution available for spherical particles, we show in the next section that expression (39) does not coincide in general with the effective electrostrictive tensor $\widetilde{\mathbf{M}}$ of a dilute suspension of aligned ellipsoidal particles.

\section{Sample results for dielectric elastomers filled with spherical particles}

In addition to their theoretical value in providing a rigorous analytical solution for the electromechanical response of dielectric elastomers with general particulate microstructures, the results (37), (38), (39) provide a formidable tool to gain insight into how the addition of particles may enhance the electromechanical properties of dielectric elastomers. In this section, we examine the specialization of the results (37), (38), (39) to the basic case of dielectric elastomers filled with an isotropic distribution of spherical particles with isotropic elastic dielectric properties. To gain further insight, we compare such results with an exact solution (Lefèvre and Lopez-Pamies, 2014) available for a random isotropic suspension of polydisperse spherical particles as well as with new full-field solutions - constructed by means of the finite-element (FE) method - for a random isotropic suspension of monodisperse spherical particles. In order to avoid loss of continuity, the details of the FE calculations are presented in Appendix A.

The practical motivation to consider isotropic distributions of spherical particles stems from recent experimental findings, including those of Zhang et al. (2002), Huang and Zhang (2004), Huang et al. (2005), Carpi and Rossi (2005), McCarthy et al. (2009), and Liu et al. (2013), which have shown that the addition of random distributions of roughly spherical particles, made up of high-permittivity or (semi)conducting solids, into dielectric elastomers leads to a drastic enhancement of the electrostrictive capabilities of these materials. In the sequel, we deploy the theoretical results (37), (38), (39) to scrutinize such findings. Furthermore, we seek to identify what other type of fillers not yet utilized in experimental studies, such as liquid-like particles with high-permittivity and vacuous pores, may potentially lead to the enhancement of the overall elastic dielectric properties of dielectric elastomers.

\subsection{The case of isotropic distributions of spherical particles with isotropic elastic dielectric properties}

For the case of spherical particles whose centers are distributed with isotropic symmetry $\mathbf{Z}=\mathbf{I}$ and the microstructural tensors (44) reduce to

$$
\left\langle\xi_{i} \xi_{j}\right\rangle=\frac{1}{3} \delta_{i j} \quad \text { and } \quad\left\langle\xi_{i} \xi_{j} \xi_{k} \xi_{l}\right\rangle=\frac{1}{15}\left(\delta_{i j} \delta_{k l}+\delta_{i k} \delta_{j l}+\delta_{i l} \delta_{j k}\right) .
$$

For this class of microstructures, assuming, for definiteness, that the particles are made up of an isotropic elastic dielectric material with modulus of elasticity, permittivity, and electrostrictive tensor of the form (40), the solutions (37), (38), (39) specialize to

$$
\widetilde{\mathbf{L}}=2 \widetilde{\mu} \mathcal{K}+3 \widetilde{\kappa} \mathcal{J}, \quad \widetilde{\boldsymbol{\epsilon}}=\widetilde{\varepsilon} \mathbf{I}, \quad \widetilde{\mathbf{M}}=\widetilde{m}_{K} \mathcal{K}+\widetilde{m}_{J} \mathcal{J},
$$


where it is recalled that the orthogonal projections tensor $\mathcal{K}$ and $\mathcal{J}$ are given by expressions (28), and

$$
\begin{aligned}
\widetilde{\mu}= & \mu+\frac{5 c(3 \kappa+4 \mu)\left(\mu_{p}-\mu\right) \mu}{[(6 c+9) \kappa+4(3 c+2) \mu] \mu+6(1-c)(\kappa+2 \mu) \mu_{p}}, \\
\widetilde{\kappa}= & \kappa+\frac{c(3 \kappa+4 \mu)\left(\kappa_{p}-\kappa\right)}{3 \kappa_{p}+4 \mu-3 c\left(\kappa_{p}-\kappa\right)}, \\
\widetilde{\varepsilon}= & \varepsilon+\frac{3 c\left(\varepsilon_{p}-\varepsilon\right) \varepsilon}{(2+c) \varepsilon+(1-c) \varepsilon_{p}}, \\
\widetilde{m}_{K}= & \varepsilon+\frac{1-c}{15 c \varepsilon} \Delta \widetilde{\varepsilon}^{2}+\frac{1-c}{15 c^{2}\left(\varepsilon_{p}-\varepsilon\right)}\left[\frac{6(3-c) \varepsilon \mu+6(1+c) \varepsilon_{p} \mu+9 \varepsilon \kappa}{(3 \kappa+4 \mu) \varepsilon \mu}+\frac{(14+c) \varepsilon+(1-c) \varepsilon_{p}}{(1-c)\left(\mu_{p}-\mu\right) \varepsilon}\right] \Delta \widetilde{\varepsilon}^{2} \Delta \widetilde{\mu}+ \\
& \frac{(1-c)(3 \kappa+8 \mu)}{5 c(3 \kappa+4 \mu) \mu} \Delta \widetilde{\varepsilon} \Delta \widetilde{\mu}, \\
\widetilde{m}_{J}= & \varepsilon+\frac{3 c\left(\varepsilon_{p}-\varepsilon\right)\left[\left(3(4 c-1) \kappa+9(1-c) \kappa_{p}+4(2+c) \mu\right) \varepsilon-(1-c)\left(6 \kappa-9 \kappa_{p}-4 \mu\right) \varepsilon_{p}\right] \varepsilon}{\left[(2+c) \varepsilon+(1-c) \varepsilon_{p}\right]^{2}\left[3 \kappa_{p}+4 \mu-3 c\left(\kappa_{p}-\kappa\right)\right]}
\end{aligned}
$$

with $\kappa=\lambda+2 \mu / 3, \kappa_{p}=\lambda_{p}+2 \mu_{p} / 3, \Delta \widetilde{\mu}=\widetilde{\mu}-\mu, \Delta \widetilde{\varepsilon}=\widetilde{\varepsilon}-\varepsilon$. The overall electromechanical constitutive response (7)-(8) for this type of isotropic dielectric elastomer composites read then simply as

$$
\begin{aligned}
\overline{\mathbf{S}}=\widetilde{\mathbf{L}} \overline{\mathbf{H}}+\widetilde{\mathbf{M}} \overline{\mathbf{E}} \otimes \overline{\mathbf{E}} & =(2 \widetilde{\mu} \mathcal{K}+3 \widetilde{\kappa} \mathcal{J}) \overline{\mathbf{H}}+\left(\widetilde{m}_{K} \mathcal{K}+\widetilde{m}_{J} \mathcal{J}\right) \overline{\mathbf{E}} \otimes \overline{\mathbf{E}} \\
& =\widetilde{\mu}\left[\overline{\mathbf{H}}+\overline{\mathbf{H}}^{T}-\frac{2}{3}(\operatorname{tr} \overline{\mathbf{H}}) \mathbf{I}\right]+\widetilde{\kappa}(\operatorname{tr} \overline{\mathbf{H}}) \mathbf{I}+\widetilde{m}_{K}\left[\overline{\mathbf{E}} \otimes \overline{\mathbf{E}}-\frac{1}{3}(\overline{\mathbf{E}} \cdot \overline{\mathbf{E}}) \mathbf{I}\right]+\frac{\widetilde{m}_{J}}{3}(\overline{\mathbf{E}} \cdot \overline{\mathbf{E}}) \mathbf{I}
\end{aligned}
$$

and

$$
\overline{\mathbf{D}}=\widetilde{\boldsymbol{\epsilon}} \overline{\mathbf{E}}=\widetilde{\varepsilon} \overline{\mathbf{E}}
$$

to leading order in the limit of small deformations and moderate electric fields.

The above results contain several limiting cases worth recording explicitly:

- Dilute volume fraction of particles. In the fundamental limit when the particles are present in dilute volume fraction as $c \rightarrow 0+$, the effective electromechanical material parameters (49) reduce asymptotically to

$$
\begin{aligned}
\widetilde{\mu} & =\mu+\frac{5(3 \kappa+4 \mu)\left(\mu_{p}-\mu\right) \mu}{(9 \kappa+8 \mu) \mu+6(\kappa+2 \mu) \mu_{p}} c, \\
\widetilde{\kappa} & =\kappa+\frac{(3 \kappa+4 \mu)\left(\kappa_{p}-\kappa\right)}{3 \kappa_{p}+4 \mu} c, \\
\widetilde{\varepsilon} & =\varepsilon+\frac{3\left(\varepsilon_{p}-\varepsilon\right) \varepsilon}{2 \varepsilon+\varepsilon_{p}} c, \\
\widetilde{m}_{K} & =\varepsilon+\frac{3\left(\varepsilon_{p}-\varepsilon\right)\left[\left(126 \kappa \mu+69 \kappa \mu_{p}+102 \mu^{2}+158 \mu \mu_{p}\right) \varepsilon+\left(9 \kappa \mu+21 \kappa \mu_{p}-42 \mu^{2}+82 \mu \mu_{p}\right) \varepsilon_{p}\right] \varepsilon}{5\left(2 \varepsilon+\varepsilon_{p}\right)^{2}\left[(9 \kappa+8 \mu) \mu+6(\kappa+2 \mu) \mu_{p}\right]} c, \\
\widetilde{m}_{J} & =\varepsilon+\frac{3\left(\varepsilon_{p}-\varepsilon\right)\left[\left(9 \kappa_{p}-3 \kappa+8 \mu\right) \varepsilon+\left(9 \kappa_{p}-6 \kappa+4 \mu\right) \varepsilon_{p}\right] \varepsilon}{\left(2 \varepsilon+\varepsilon_{p}\right)^{2}\left(3 \kappa_{p}+4 \mu\right)} c
\end{aligned}
$$

up to $O(c)$.

- Rigid particles with infinite permittivity. In the limit of rigid infinite-permittivity particles when $\mu_{p}, \kappa_{p}=+\infty$ and $\varepsilon_{p}=+\infty$, the effective electromechanical material parameters (49) reduce to

$$
\begin{aligned}
& \tilde{\mu}=\mu+\frac{5 c(3 \kappa+4 \mu)}{6(1-c)(\kappa+2 \mu)} \mu, \quad \widetilde{\kappa}=\kappa+\frac{c(3 \kappa+4 \mu)}{3(1-c)}, \quad \widetilde{\varepsilon}=\varepsilon+\frac{3 c}{1-c} \varepsilon, \\
& \tilde{m}_{K}=\varepsilon+\frac{c[21(1-c) \kappa+(82-22 c) \mu]}{10(1-c)^{2}(\kappa+2 \mu)} \varepsilon, \quad \widetilde{m}_{J}=\varepsilon+\frac{9 c}{(1-c)^{2}} \varepsilon .
\end{aligned}
$$


If, in addition, the underlying elastomeric matrix is incompressible $(\kappa=+\infty)$, these formulae reduce further to

$$
\begin{aligned}
& \widetilde{\mu}=\mu+\frac{5 c}{2(1-c)} \mu, \quad \widetilde{\kappa}=+\infty, \quad \widetilde{\varepsilon}=\varepsilon+\frac{3 c}{1-c} \varepsilon, \\
& \widetilde{m}_{K}=\varepsilon+\frac{21 c}{10(1-c)} \varepsilon, \quad \widetilde{m}_{J}=\varepsilon+\frac{9 c}{(1-c)^{2}} \varepsilon .
\end{aligned}
$$

The results (53)-(54) are relevant for dielectric elastomer composites wherein the filler particles are typical ceramics (e.g., titania) or metals (e.g., iron), which generally exhibit much larger stiffness and permittivity (infinitely larger for the case of metals) than elastomers.

- Liquid particles with infinite permittivity. The case $\mu_{p}=0, \kappa_{p}=+\infty$, and $\varepsilon_{p}=+\infty$ corresponds to particles that are liquid-like (incompressible with vanishingly small shear modulus) and of infinite permittivity. Granted these limiting values for the properties of the particles, the effective electromechanical material parameters (49) reduce to

$$
\begin{aligned}
& \widetilde{\mu}=\mu-\frac{5 c(3 \kappa+4 \mu)}{(9+6 c) \kappa+4(2+3 c) \mu} \mu, \quad \widetilde{\kappa}=\kappa+\frac{c(3 \kappa+4 \mu)}{3(1-c)}, \quad \widetilde{\varepsilon}=\varepsilon+\frac{3 c}{1-c} \varepsilon, \\
& \widetilde{m}_{K}=\varepsilon+\frac{3 c[(9+21 c) \kappa-(42-22 c) \mu]}{5(1-c)[(9+6 c) \kappa+4(2+3 c) \mu]} \varepsilon, \quad \widetilde{m}_{J}=\varepsilon+\frac{9 c}{(1-c)^{2}} \varepsilon .
\end{aligned}
$$

When the underlying elastomeric matrix is incompressible $(\kappa=+\infty)$, these formulae reduce further to

$$
\begin{aligned}
& \widetilde{\mu}=\mu-\frac{5 c}{3+2 c} \mu, \quad \widetilde{\kappa}=+\infty, \quad \widetilde{\varepsilon}=\varepsilon+\frac{3 c}{1-c} \varepsilon, \\
& \widetilde{m}_{K}=\varepsilon+\frac{3 c(3+7 c)}{5(1-c)(3+2 c)} \varepsilon, \quad \widetilde{m}_{J}=\varepsilon+\frac{9 c}{(1-c)^{2}} \varepsilon .
\end{aligned}
$$

Compared with elastomers, many fluids (e.g., water) and eutectic alloys (e.g., Galinstan) can be idealized as incompressible and as having zero shear modulus and infinite permittivity (see, e.g., Wang et al., 2012; Fassler and Majidi, 2015). Expressions (55)-(56) are relevant for such type of filler particles.

- Porous dielectric elastomers. The limiting values $\mu_{p}, \kappa_{p}=0, \varepsilon_{p}=\varepsilon_{0}, \kappa=+\infty$, where $\varepsilon_{0}$ stands for the permittivity of vacuum, correspond to an incompressible matrix containing an isotropic distribution of vacuous pores. In this case, the effective electromechanical material parameters (49) reduce to

$$
\begin{aligned}
& \widetilde{\mu}=\mu-\frac{5 c}{3+2 c} \mu, \quad \widetilde{\kappa}=\frac{4(1-c)}{3 c} \mu, \quad \widetilde{\varepsilon}=\varepsilon+\frac{3 c\left(\varepsilon_{0}-\varepsilon\right) \varepsilon}{(2+c) \varepsilon+(1-c) \varepsilon_{0}}, \\
& \widetilde{m}_{K}=\varepsilon-\frac{3 c\left(\varepsilon-\varepsilon_{0}\right)\left[(c(7 c+26)+42) \varepsilon+(c(4-7 c)+3) \varepsilon_{0}\right]}{5(3+2 c)\left[(2+c) \varepsilon+(1-c) \varepsilon_{0}\right]^{2}} \varepsilon, \\
& \widetilde{m}_{J}=\varepsilon-\frac{3\left(\varepsilon-\varepsilon_{0}\right)\left[(4 c-1) \varepsilon-2(1-c) \varepsilon_{0}\right]}{\left[(2+c) \varepsilon+(1-c) \varepsilon_{0}\right]^{2}} \varepsilon .
\end{aligned}
$$

\subsubsection{Electrostriction}

In the absence of applied stresses when $\overline{\mathbf{S}}=\mathbf{0}$, it follows from the overall constitutive relation (50) that

$$
\overline{\mathbf{H}}=-\frac{\widetilde{m}_{K}}{2 \widetilde{\mu}} \overline{\mathbf{E}} \otimes \overline{\mathbf{E}}+\left[\frac{\widetilde{m}_{K}}{6 \widetilde{\mu}}-\frac{\widetilde{m}_{J}}{9 \widetilde{\kappa}}\right](\overline{\mathbf{E}} \cdot \overline{\mathbf{E}}) \mathbf{I} .
$$

The deformation measure (58) is referred to as the electrostriction that the dielectric elastomer composite undergoes when it is subjected to a macroscopic electric field $\overline{\mathbf{E}}$. 
In practice, it is often the case that just a uniaxial electric field is applied to probe the electrostriction of deformable dielectrics. This is commonly accomplished by sandwiching a thin layer of the material in between two compliant electrodes connected to a battery. For such a configuration, the macroscopic stress is indeed roughly zero everywhere (inside the material as well as in the surrounding space), while the macroscopic electric field is roughly uniform within the material and zero outside of it. For an applied uniaxial electric field of the form

$$
\overline{\mathbf{E}}=\bar{E} \mathbf{e}_{3}
$$

with $\bar{E}=\Phi / L_{3}$, where $\Phi$ denotes the voltage applied between the electrodes and $L_{3}$ stands for the initial thickness of the thin layer of dielectric elastomer composite, the electrostriction (58) takes the diagonal form

$$
\overline{\mathbf{H}}=\bar{H}_{11} \mathbf{e}_{1} \otimes \mathbf{e}_{1}+\bar{H}_{22} \mathbf{e}_{2} \otimes \mathbf{e}_{2}+\bar{H}_{33} \mathbf{e}_{3} \otimes \mathbf{e}_{3},
$$

where

$$
\bar{H}_{11}=\bar{H}_{22}=\left(\frac{\widetilde{m}_{K}}{6 \widetilde{\mu}}-\frac{\widetilde{m}_{J}}{9 \widetilde{\kappa}}\right) \bar{E}^{2} \quad \text { and } \quad \bar{H}_{33}=-\left(\frac{\widetilde{m}_{K}}{3 \widetilde{\mu}}+\frac{\widetilde{m}_{J}}{9 \widetilde{\kappa}}\right) \bar{E}^{2} .
$$

Figure 2 shows a schematic of this thought experiment with the various quantities of interest indicated.

(a)

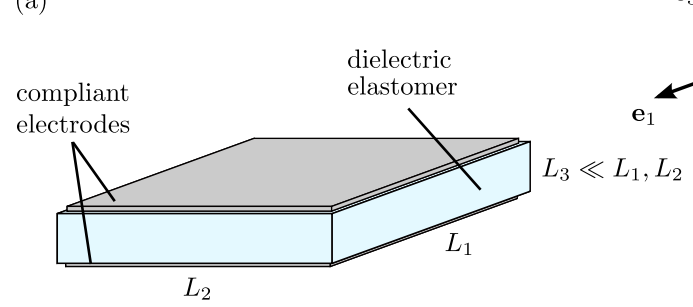

(b)

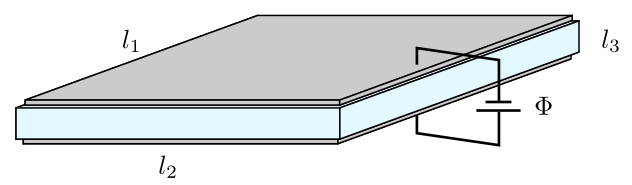

Figure 2: Schematic of the typical experimental setup — in (a) the undeformed and (b) the deformed configurations - to probe the electrostriction of dielectric elastomers under the application of a uniaxial electric field (59).

For later reference, we note that in the absence of particles $(c=0)$ expressions $(61)$ reduce to

$$
H_{11}^{m}=H_{22}^{m}=\left(\frac{\varepsilon}{6 \mu}-\frac{\varepsilon}{9 \kappa}\right) \bar{E}^{2} \quad \text { and } \quad H_{33}^{m}=-\left(\frac{\varepsilon}{3 \mu}+\frac{\varepsilon}{9 \kappa}\right) \bar{E}^{2},
$$

the electrostriction in the transverse and thickness directions of the pure (unfilled) elastomeric matrix. The ratios

$$
\frac{\bar{H}_{11}}{H_{11}^{m}}=\frac{\bar{H}_{22}}{H_{22}^{m}}=\frac{\frac{\widetilde{m}_{K}}{2 \widetilde{\mu}}-\frac{\widetilde{m}_{J}}{3 \widetilde{\kappa}}}{\frac{\varepsilon}{2 \mu}-\frac{\varepsilon}{3 \kappa}} \quad \text { and } \quad \frac{\bar{H}_{33}}{H_{33}^{m}}=\frac{\frac{\tilde{m}_{K}}{\widetilde{\mu}}+\frac{\widetilde{m}_{J}}{3 \widetilde{\kappa}}}{\frac{\varepsilon}{\mu}+\frac{\varepsilon}{3 \kappa}}
$$

provide then direct insight into how the addition of particles affects the electrostriction of dielectric elastomers.

\subsubsection{Comparison with an exact solution for a suspension of polydisperse spherical particles}

In a recent contribution, Lefèvre and Lopez-Pamies (2014) have worked out an exact solution for the overall elastic dielectric response of a special class of isotropic suspensions of spherical particles in rubber, the so-called differential coated sphere (DCS) assemblage (see, e.g., Avellaneda, 1987; Chapter 10.5 in Milton, 2002). Roughly speaking, this class of suspensions comprises spherical particles of infinitely many sizes distributed in such a way that particles of any given comparable size are far apart from each other and surrounded by particles of much smaller size. For the case of interest here, when the elastomeric matrix material and the particles are characterized by relations (25) and (40), the effective shear modulus, bulk 
modulus, permittivity, and electrostrictive coefficients of such suspensions read as

$$
\begin{aligned}
\widetilde{\mu}^{\mathrm{DCS}}= & \frac{q_{2}+\sqrt{q_{2}^{2}+4 q_{1} q_{3}}}{2 q_{1}} \mu, \\
\widetilde{\kappa}^{\mathrm{DCS}}= & \kappa+\frac{c(3 \kappa+4 \mu)\left(\kappa_{p}-\kappa\right)}{3 \kappa_{p}+4 \mu-3 c\left(\kappa_{p}-\kappa\right)}, \\
\widetilde{\varepsilon}^{\mathrm{DCS}}= & \varepsilon+\frac{3 c\left(\varepsilon_{p}-\varepsilon\right) \varepsilon}{(2+c) \varepsilon+(1-c) \varepsilon_{p}}, \\
\widetilde{m}_{K}^{\mathrm{DCS}}= & \frac{2\left(\widetilde{\varepsilon}^{\mathrm{DCS}}-\varepsilon\right)^{2} \varepsilon_{p}\left[A_{1}+\frac{21 c^{2 / 3}\left(3 \kappa_{p}+\mu_{p}\right)}{5\left(15 \kappa_{p}+11 \mu_{p}\right)} A_{3}\right]}{c\left(\varepsilon_{p}-\varepsilon\right)^{2}}+ \\
& \frac{2 B_{1}(1-c)\left(\widetilde{\varepsilon}^{\mathrm{DCS}}-\varepsilon\right)^{2}\left[(20+c) \varepsilon^{2}+2(10-c) \varepsilon \varepsilon_{p}+(5+c) \varepsilon_{p}^{2}\right]}{45 c^{2} \varepsilon\left(\varepsilon_{p}-\varepsilon\right)^{2}}-\frac{3 B_{2}\left(1-\frac{1}{c^{8 / 3}}\right)\left(\widetilde{\varepsilon}^{\mathrm{DCS}}-\varepsilon\right)^{2}}{5 \varepsilon}- \\
& \frac{2 B_{3}\left(\widetilde{\varepsilon}^{\mathrm{DCS}}-\varepsilon\right)^{2}\left[\frac{7\left(c^{5 / 3}-1\right)\left(2 \varepsilon+\varepsilon_{p}\right)^{2}(3 \kappa+\mu)}{c^{5 / 3}\left(\varepsilon_{p}-\varepsilon\right)^{2}}+\frac{7\left(1-c^{2 / 3}\right)\left(2 \varepsilon+\varepsilon_{p}\right)(8 \mu-3 \kappa)}{c^{2 / 3}\left(\varepsilon_{p}-\varepsilon\right)}+\left(c^{1 / 3}-1\right)(15 \kappa-31 \mu)\right]}{15 c^{1 / 3} \varepsilon(15 \kappa+11 \mu)}+ \\
& \frac{B_{4}(1-c)\left(\widetilde{\varepsilon}^{\mathrm{DCS}}-\varepsilon\right)^{2}\left[(1+5 c) \kappa \varepsilon-2(7-c) \mu \varepsilon-(7+5 c) \kappa \varepsilon_{p}-2(5+c) \mu \varepsilon_{p}\right]}{15 c^{2} \varepsilon \mu\left(\varepsilon_{p}-\varepsilon\right)}, \\
\widetilde{m}_{J}^{\mathrm{DCS}}= & \varepsilon+\frac{3 c\left(\varepsilon_{p}-\varepsilon\right)\left[\left(3(4 c-1) \kappa+9(1-c) \kappa_{p}+4(2+c) \mu\right) \varepsilon-(1-c)\left(6 \kappa-9 \kappa_{p}-4 \mu\right) \varepsilon_{p}\right] \varepsilon}{\left[(2+c) \varepsilon+(1-c) \varepsilon_{p}\right]^{2}\left[3 \kappa_{p}+4 \mu-3 c\left(\kappa_{p}-\kappa\right)\right]}, \quad(64)
\end{aligned}
$$

where the two sets of parameters $q_{1}, q_{2}, q_{3}$ and $A_{1}, A_{3}, B_{1}, B_{2}, B_{3}, B_{4}$ are defined, respectively, in Appendices II and I in Lefèvre and Lopez-Pamies (2014).

Remarkably, the results $(49)_{2},(49)_{3}$, and $(49)_{5}$ for the effective bulk modulus $\widetilde{\kappa}$, effective permittivity $\widetilde{\varepsilon}$, and effective electrostrictive coefficient $\widetilde{m}_{J}$ are seen to agree identically with the corresponding effective material parameters $(64)_{2},(64)_{3}$, and $(64)_{5}$ for a suspension of polydisperse spherical particles. By contrast, the results $(49)_{1}$ and $(49)_{4}$ for the effective shear modulus $\widetilde{\mu}$ and effective electrostrictive coefficient $\widetilde{m}_{K}$ differ in general from expressions $(64)_{1}$ and $(64)_{4}$. In the dilute limit of particles as $c \rightarrow 0+$, these latter expressions reduce asymptotically to

$$
\begin{aligned}
\widetilde{\mu}^{\mathrm{DCS}} & =\mu+\frac{5(3 \kappa+4 \mu)\left(\mu_{p}-\mu\right) \mu}{(9 \kappa+8 \mu) \mu+6(\kappa+2 \mu) \mu_{p}} c, \\
\widetilde{m}_{K}^{\mathrm{DCS}} & =\varepsilon+\frac{3\left(\varepsilon_{p}-\varepsilon\right)\left[\left(27 \kappa \mu+12 \kappa \mu_{p}+21 \mu^{2}+31 \mu \mu_{p}\right) \varepsilon+\left(6 \kappa \mu_{p}-9 \mu^{2}+17 \mu \mu_{p}\right) \varepsilon_{p}\right] \varepsilon}{\left(2 \varepsilon+\varepsilon_{p}\right)^{2}\left[(9 \kappa+8 \mu) \mu+6(\kappa+2 \mu) \mu_{p}\right]} c
\end{aligned}
$$

up to $O(c)$. Thus, as anticipated in remark $i v$ of Section 4 , the result $(49)_{1}$ does agree identically with the exact effective shear modulus $(65)_{1}$ for a dilute suspension of spherical particles $\left(c f\right.$. expression $\left.(52)_{1}\right)$, but the same is not true for the result $(49)_{4}$, whose asymptotic form $(52)_{4}$ in the dilute limit is in general different from the effective electrostrictive coefficient $(65)_{2}$.

The above comparison reveals that the effective electromechanical material parameters (49), even though exact for a different class of two-phase particulate isotropic microstructures, serve as well to describe approximately in general, but exactly in some special cases - the overall elastic dielectric response of a random isotropic suspension of polydisperse spherical particles. In this regard, we emphasize again that the microstructure for which the effective constants (49) are exact was selected to have the same one- and two-point correlation functions as an isotropic suspension of spherical particles; see remark $i i$ of Section 4 . In the sequel, we present further comparisons between the theoretical results (49) and full-field simulations for a random isotropic suspension of monodisperse spherical particles.

\subsection{Results for stiff particles with high permittivity}

We begin by examining the case of dielectric elastomer composites wherein the filler particles are mechanically stiffer than the elastomeric matrix and also exhibit higher permittivity. As pointed out above, this 
is the case that has hitherto received most attention by the experimental community, presumably because most filler materials with high permittivity or (semi-)conducting behavior (e.g., ceramics, metals) are stiffer than elastomers.

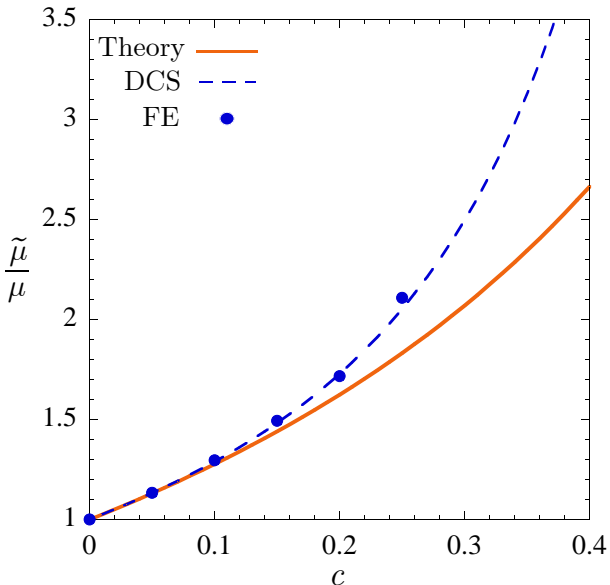

(a)

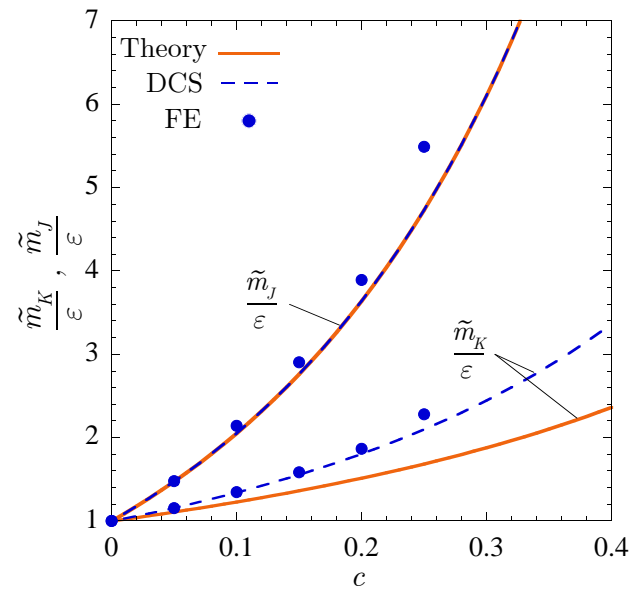

(c)

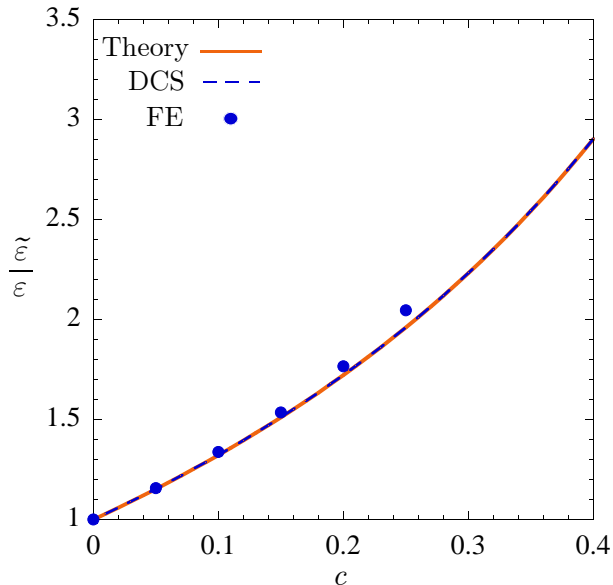

(b)

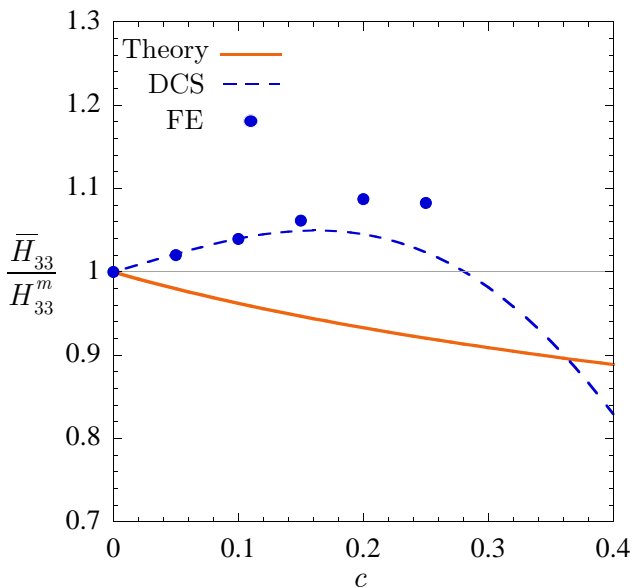

(d)

Figure 3: Overall electromechanical response of a nearly incompressible dielectric elastomer $\left(\kappa / \mu=10^{3}\right)$ filled with a random isotropic distribution of stiff high-permittivity spherical particles $\left(\mu_{p} / \mu=10^{5}, \kappa_{p} / \mu=10^{5}, \varepsilon_{p} / \varepsilon=10^{2}\right)$. Results are shown for: (a) the normalized effective shear modulus $\widetilde{\mu} / \mu$, (b) the normalized effective permittivity $\widetilde{\varepsilon} / \varepsilon$, (c) the normalized effective electrostrictive coefficients $\widetilde{m}_{K} / \varepsilon, \widetilde{m}_{J} / \varepsilon$, and (d) the ratio of electrostrictions $\bar{H}_{33} / H_{33}^{m}$, all as functions of the volume fraction of particles $c$. The solid lines in the plots correspond to the theoretical results (49). The dashed lines and solid circles stand for corresponding results based on the analytical solution (64) for an isotropic suspension of polydisperse spherical particles and on FE simulations for an isotropic suspension of monodisperse spherical particles.

Figures 3(a), (b), (c) show results for the normalized effective shear modulus $\widetilde{\mu} / \mu$, permittivity $\widetilde{\varepsilon} / \varepsilon$, and electrostrictive coefficients $\widetilde{m}_{K} / \varepsilon, \widetilde{m}_{J} / \varepsilon$ of a dielectric elastomer composite comprised of a nearly incompressible elastomeric matrix with $\kappa / \mu=10^{3}$ and stiff high-permittivity filler particles with $\mu_{p} / \mu=10^{5}$, $\kappa_{p} / \mu=10^{5}$, and $\varepsilon_{p} / \varepsilon=10^{2}$, as functions of the volume fraction of particles $c$ (no results for the effective bulk modulus $\widetilde{\kappa}$ are included since the overall response is nearly incompressible in this case). The rationale behind this choice of material parameters is that they are typical of many of the dielectric elastomer composites studied experimentally, such as for instance those of Liu et al. (2013), where the elastomer is silicone rubber $\left(\mu=0.22 \mathrm{MPa}, \kappa=1 \mathrm{GPa}, \varepsilon=3.2 \varepsilon_{0}\right)$ and the particles are made out of titania $\left(\mu_{\mathrm{p}}=110\right.$ $\left.\mathrm{GPa}, \kappa_{\mathrm{p}}=220 \mathrm{GPa}, \varepsilon_{\mathrm{p}}=114 \varepsilon_{0}\right)$. In these and subsequent figures, the solid lines are associated with the theoretical results (49), while the dashed lines and solid circles stand for corresponding results based on the 
analytical solution (64) for isotropic suspensions of polydisperse spherical particles and on FE simulations for isotropic suspensions of monodisperse spherical particles.

As discussed in Section 5.1.2, Figs. 3(a), (b), (c) illustrate that the theoretical results are in fairly good agreement with the results for suspensions of polydisperse and also monodisperse spherical particles, save quantitatively for the effective shear modulus $\widetilde{\mu}$ when $c>0.15$ and for the effective electrostrictive coefficient $\widetilde{m}_{K}$ when $c>0.05$. Another immediate observation from Figs. 3(a) and (b) is that the addition of stiff high-permittivity particles enhances both the stiffness and permittivity of the dielectric elastomer. Figure $3(\mathrm{c})$ shows that this is also the case for the electrostrictive coefficients $\widetilde{m}_{K}$ and $\widetilde{m}_{J}$. According to equation (58), and as expected on physical grounds, these trends set up a direct competition of effects for the overall electrostriction capabilities of the composite. To see which enhancement proves dominant, if the enhancement in stiffness (which makes the material less deformable) or that in permittivity and electrostrictive coefficients (which makes the material more prone to deform under the application of an electric field), we turn to examine the behavior of the ratio of electrostrictions $\bar{H}_{33} / H_{33}^{m}$ associated with the response of the composite under a uniaxial electric field; see Section 5.1.1.

Figure $3\left(\mathrm{~d}\right.$ ) shows plots of the ratio $\bar{H}_{33} / H_{33}^{m}$ as a function of the volume fraction of particles $c$ (no results for the ratio $\bar{H}_{11} / H_{11}^{m}$ are shown here since the overall near incompressibility of the composite implies that $\left.\bar{H}_{11} / H_{11}^{m} \approx \bar{H}_{33} / H_{33}^{m}\right)$. The theoretical results indicate that $\bar{H}_{33} / H_{33}^{m}<1$, that is, the addition of particles leads to a reduction in electrostriction. Physically, this entails that the enhancement in stiffness due to the addition of particles dominates over the enhancement in permittivity resulting in the filled elastomer undergoing less electrostriction than the unfilled elastomer when exposed to the same electric field. This behavior is in contrast to that displayed by the suspensions of spherical particles, which initially and up to about $c=0.3$ exhibit an enhancement in electrostriction $\left(\bar{H}_{33} / H_{33}^{m}>1\right)$ before displaying a reduction $\left(\bar{H}_{33} / H_{33}^{m}<1\right)$.

While qualitatively different, all three sets of results in Fig. 3(d) agree in that the reduction or enhancement is quantitatively small, indeed $0.8<\bar{H}_{33} / H_{33}^{m}<1.15$ for the entire range of particle volume fractions considered, $0 \leq c \leq 0.4$. Such a difference in qualitative behavior but agreement in quantitative behavior among three different exact results for three different two-phase particulate isotropic microstructures suggest that the electrostriction capabilities of dielectric elastomers filled isotropically with stiff high-permittivity particles is highly sensitive to the details of the microstructure, but only in a qualitative manner. Quantitatively, moreover, they suggest that the enhancement in stiffness provided by the addition of filler particles essentially cancels out the enhancement in permittivity, so that there is ultimately little difference between the electrostriction capabilities of the unfilled and the filled elastomer (for particle volume fractions sufficiently away from percolation, of course).

Having gained insight into the theoretical results (49) for the case of stiff high-permittivity filler particles, we now deploy them to scrutinize two representative experiments: $i$ ) the experiments of Liu et al. (2013) for the electrostriction of a silicone (PDMS) elastomer filled with titania $\left(\mathrm{TiO}_{2}\right)$ particles and $\left.i i\right)$ the experiments of Huang et al. (2005) for the electrostriction of a polyurethane (PU) elastomer filled with semi-conductor copper pthalocyanine oligomer (o-CuPc) particles, coated by a polyacrylic acid (PAA), under the application of a uniaxial electric field.

The material parameters describing the elastic dielectric response of the PDMS elastomer utilized by Liu et al. (2013) are approximately given by $\mu=0.22 \mathrm{MPa}, \kappa=1 \mathrm{GPa}, \varepsilon=3.2 \varepsilon_{0}$, while those of the $\mathrm{TiO}_{2}$ particles are $\mu_{p}=110 \mathrm{GPa}, \kappa_{p}=220 \mathrm{GPa}, \varepsilon_{p}=114 \varepsilon_{0}$. The particles were reported to be roughly spherical in shape, about $3 \mu \mathrm{m}$ in average diameter, and spatially well dispersed throughout the PDMS matrix. Figure 4(a) shows the experimentally measured electrostriction $\bar{H}_{11}$ in the transverse direction for the pure PDMS elastomer (solid triangles) and the PDMS elastomer filled with a volume fraction $c=0.082$ of $\mathrm{TiO}_{2}$ particles (hollow triangles), as functions of the magnitude of the applied electric field $\bar{E}$. The dotted and solid lines correspond to the theoretical predictions for the electrostriction of the unfilled $(c=0)$ and the filled $(c=0.082)$ PDMS elastomer, respectively, based on the result $(61)_{1}$ with (49). The theoretical prediction for the filled PDMS elastomer based on the analytical solution (64) for an isotropic suspension of polydisperse spherical particles is also included in the figure (dashed line) for comparison purposes.

On the other hand, the material parameters describing the elastic dielectric response of the PU elastomer utilized by Huang et al. (2005) are approximately given by $\mu=9 \mathrm{MPa}, \kappa=5 \mathrm{GPa}, \varepsilon=8 \varepsilon_{0}$, while those 


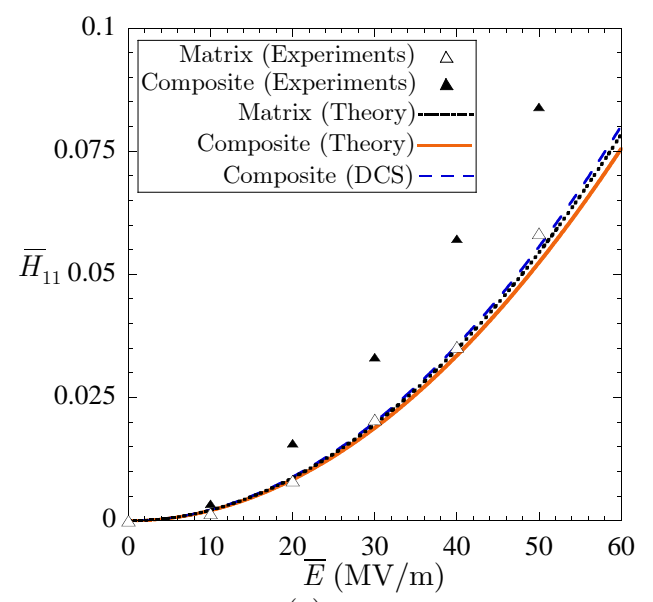

(a)

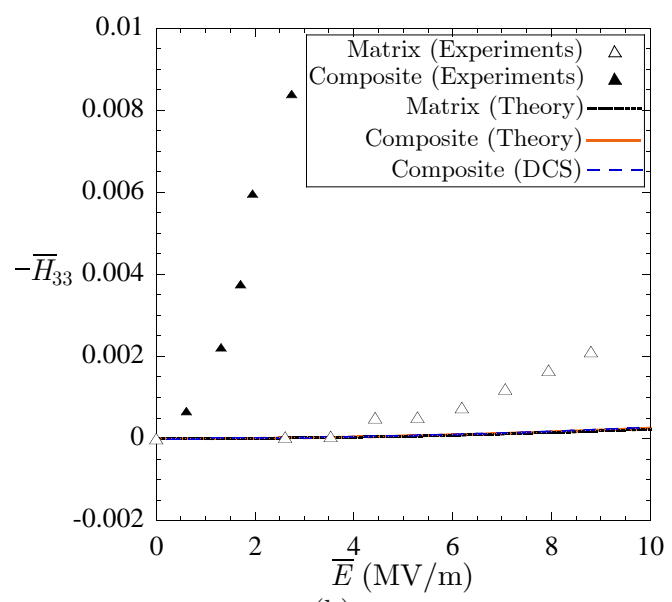

(b)

Figure 4: Comparisons between the theoretical results and the experimental data of Liu et al. (2013) and Huang et al. (2005) for the electrostriction of: (a) a PDMS elastomer filled with $\mathrm{TiO}_{2}$ particles at volume fraction $c=0.082$ and (b) a PU elastomer filled with o-CuPc particles at volume fraction $c=0.073$, under the application of a uniaxial electric field with magnitude $\bar{E}$. The experimental data for the filled elastomers is depicted as solid triangles, while the data for the pure (unfilled) elastomers is depicted as hollow triangles. The dotted and solid lines correspond to the theoretical predictions (61) with (49) for the pure and the filled elastomers, respectively. The dashed lines stand for the predictions based on the analytical solution (64) for an isotropic suspension of polydisperse spherical particles.

of the o-CuPc filler particles are $\mu_{p}=1 \mathrm{GPa}, \kappa_{p}=100 \mathrm{GPa}, \varepsilon_{p}=10^{4} \varepsilon_{0}$. In this case too, the particles were roughly spherical in shape, about $40 \mathrm{~nm}$ in diameter, and spatially well dispersed throughout the PU matrix. The volume fraction of the particles was reported to be approximately $c=0.073$. Figure 4 (b) shows the experimentally measured electrostriction $\bar{H}_{33}$ in the thickness direction for the pure PU elastomer (solid triangles) and the filled PU elastomer (hollow triangles), as functions of the magnitude of the applied electric field $\bar{E}$. Similar to Fig. 4(a), the dotted and solids lines correspond to the theoretical predictions $(61)_{2}$ with (49) for the pure $(c=0)$ and the filled $(c=0.073)$ PU elastomer, while the dashed line corresponds to the theoretical prediction for the filled PU elastomer based on the analytical solution (64).

It is apparent from Fig. 4(a) that the response of the filled PDMS elastomer exhibits a significant enhancement in electrostriction, in the order of $50 \%$ increase, when compared with the pure PDMS elastomer. In disaccord with this experimentally observed enhancement, as already discussed within the context of Fig. $3(\mathrm{~d})$, the theoretical predictions show little change between the electrostriction of the pure and the filled PDMS elastomers. Fig. 4(b) shows an even more glaring difference between the experimental response of the filled PU elastomer, which exhibits about a 20 -fold enhancement in electrostriction compared to the pure PU elastomer, and the theoretical predictions. This dramatic difference occurs consistently for the entire range of deformations $\bar{H}_{33}$ and electric fields $\bar{E}$ considered, including small values of $\bar{H}_{33}$ and $\bar{E}$ for which the asymptotic premise of "small" deformations and "moderate" electric fields - upon which the formulas (61) and (49) are based - is expected to be applicable.

We conjecture that the drastic electrostriction enhancement observed in the experiments may be due to the presence of high-permittivity interphases and/or interphasial free charges around the filler particles, which the proposed theory does not account for. Indeed, it is well known that in elastomers filled with stiff particles the "anchoring" of the polymeric chains of the matrix onto the particles forces the chains into conformations that are very different from those in the bulk, hence resulting in "interphases" of possibly several tens of nanometers in thickness that have very different mechanical and physical properties from those in the bulk (see, e.g., Lewis, 2004; Roy et al., 2005; Leblanc, 2010; Goudarzi et al., 2015 and references therein). Furthermore, free charges in such interphases may be present from the outset because of the fabrication process of the specimens. They may also be injected from the particles upon the application of an electric field. In this regard, we note that in the experiments of Huang et al. (2005) the particles 
were made out of an organic semi-conductor coated by an anionic polyacrylic acid. Whatever their origin, the presence of interphasial charges has been recently shown to have the potential to lead to extreme enhancements of the overall dielectric response of particulate composites and, by the same token, extreme enhancements of their electrostrictive response (Lopez-Pamies et al., 2014).

\subsection{Results for liquid-like particles with high permittivity}

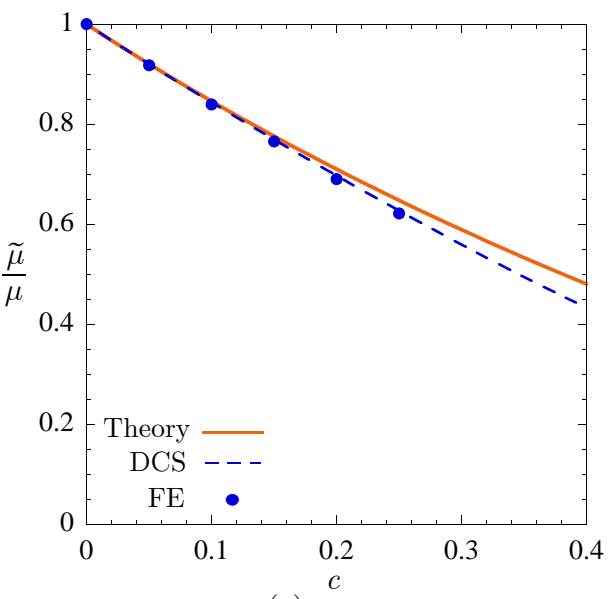

(a)

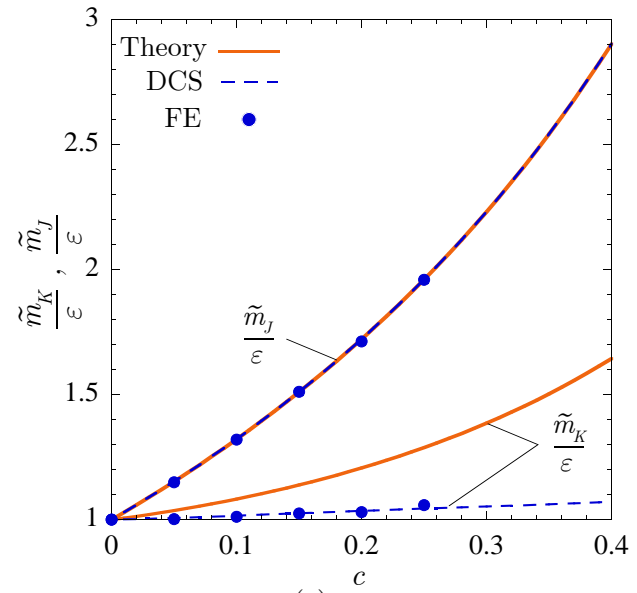

(c)

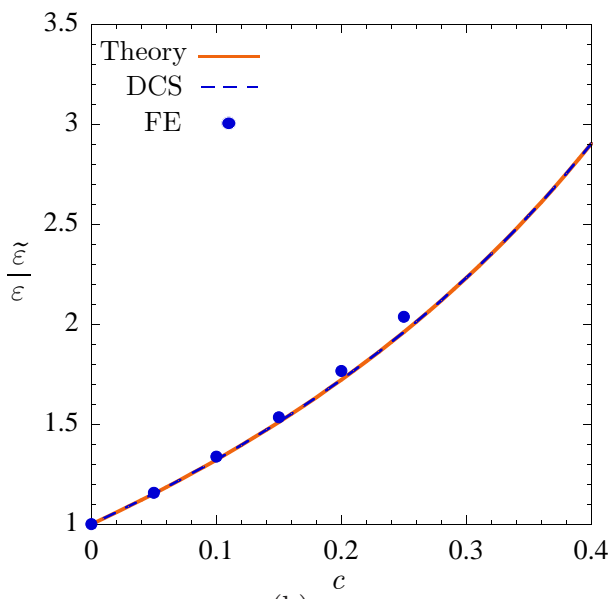

(b)

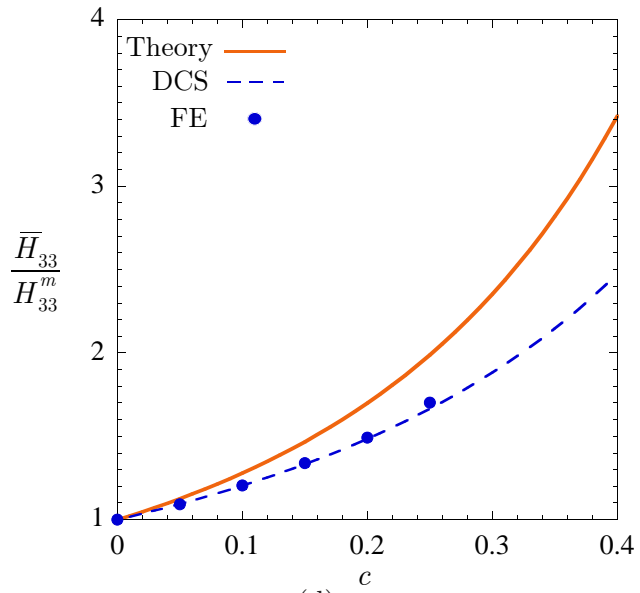

(d)

Figure 5: Overall electromechanical response of a nearly incompressible dielectric elastomer $\left(\kappa / \mu=10^{3}\right)$ filled with a random isotropic distribution of liquid-like high-permittivity spherical particles $\left(\mu_{p} / \mu=10^{-2}, \kappa_{p} / \mu=10^{3}, \varepsilon_{p} / \varepsilon=10^{2}\right)$. Results are shown for: (a) the normalized effective shear modulus $\widetilde{\mu} / \mu$, (b) the normalized effective permittivity $\widetilde{\varepsilon} / \varepsilon$, (c) the normalized effective electrostrictive coefficients $\widetilde{m}_{K} / \varepsilon, \widetilde{m}_{J} / \varepsilon$, and (d) the ratio of electrostrictions $\bar{H}_{33} / H_{33}^{m}$, all as functions of the volume fraction of particles $c$. The solid lines in the plots correspond to the theoretical results (49). The dashed lines and solid circles stand for corresponding results based on the analytical solution (64) for an isotropic suspension of polydisperse spherical particles and on FE simulations for an isotropic suspension of monodisperse spherical particles.

Next, we examine the case of dielectric elastomer composites wherein the filler particles are liquid-like, in the sense that they are characterized by nearly incompressible behavior and vanishingly small shear stiffness, and exhibit a higher permittivity than the elastomeric matrix. Such properties are distinctive of many common fluids such as water and special types of alloys such as Galistan. The practical interest in this type of dielectric elastomer composites is that increasing the content of their fillers increases the overall permittivity at the same time that it also increases the overall deformability (in contrast to the 
mechanically stiff particles considered in the preceding subsection) and thus has the potential to bestow the resulting composites with exceptionally enhanced electrostriction capabilities.

Figure $5(\mathrm{a}),(\mathrm{b}),(\mathrm{c})$ show results for the normalized effective shear modulus $\widetilde{\mu} / \mu$, permittivity $\widetilde{\varepsilon} / \varepsilon$, and electrostrictive coefficients $\widetilde{m}_{K} / \varepsilon, \widetilde{m}_{J} / \varepsilon$ of a dielectric elastomer composite comprised of a nearly incompressible elastomeric matrix with $\kappa / \mu=10^{3}$ and liquid-like high-permittivity filler particles with $\mu_{p} / \mu=10^{-2}$, $\kappa_{p} / \mu=10^{3}$, and $\varepsilon_{p} / \varepsilon=10^{2}$, as functions of the volume fraction of particles $c$. Figure $5(\mathrm{~d})$ shows the associated ratio of electrostrictions $\bar{H}_{33} / \bar{H}_{33}^{m}$ also as a function of $c$.

Consistent with the preceding case for mechanically stiff particles, the theoretical results (49) are seen to be in fairly good agreement with the results for suspensions of polydisperse and monodisperse spherical particles, save quantitatively for the effective electrostrictive coefficient $\widetilde{m}_{K}$. As expected on physical grounds, Figs. 5(a) and (b) confirm that the addition of liquid-like high-permittivity particles decreases the overall shear stiffness but increases the overall permittivity of the composite. Figure $5(\mathrm{c})$ shows that the effective electrostrictive coefficients $\widetilde{m}_{K}$ and $\widetilde{m}_{J}$ increase also with the addition of such fillers. This monotonic decrease in stiffness together with the increase in permittivity and electrostrictive coefficients entail that the electrostriction capabilities of the resulting dielectric elastomer composite are enhanced with the addition of fillers. This is precisely what is shown by Fig. $5(\mathrm{~d})$, which illustrates not only that indeed $\bar{H}_{33} / H_{33}^{m}>1$ for all $c$ but also reveals that more than a $50 \%$ enhancement in electrostriction can be achieved with the addition of a moderate content of liquid-like high-permittivity particles. It would be interesting to explore these encouraging findings experimentally.

\subsection{Results for porous dielectric elastomers}

Finally, we consider the overall electromechanical response of porous dielectric elastomers made up of a dielectric elastomer containing a random isotropic distribution of vacuous spherical pores. Here, it is important to recognize that vacuous pores are mechanically softer at the same time that they exhibit lower permittivity than elastomers. Thus their addition results in an increase in overall deformability but also a decrease in overall permittivity setting up — similar to the case of stiff high-permittivity fillers — a direct competition of effects for the overall electrostriction capabilities of the composite.

Figure 6 presents the effect that the addition of vacuous pores $\left(\mu_{p}=0, \kappa_{p}=0, \varepsilon_{p}=\varepsilon_{0}\right)$ has on the normalized effective shear modulus $\widetilde{\mu} / \mu$, bulk modulus $\widetilde{\kappa} / \kappa$, permittivity $\widetilde{\varepsilon} / \varepsilon$, and electrostrictive coefficients $\widetilde{m}_{K} / \varepsilon, \widetilde{m}_{J} / \varepsilon$ to a nearly incompressible dielectric elastomer with bulk modulus $\kappa / \mu=10^{3}$ and permittivity ${ }^{2}$ $\varepsilon / \varepsilon_{0}=3$.

Similar to the two preceding types of dielectric elastomer composites with high-permittivity fillers, the theoretical results (49) exhibit good agreement with the results for suspensions of polydisperse and mondisperse spherical pores. Figures 6(a)-(b) illustrate that the isotropic addition of vacuous spherical pores reduces significantly the shear stiffness and permittivity of the dielectric elastomer and reduces drastically its volumetric stiffness leading to a highly compressible overall behavior. Figure 6(c) indicates that the electrostrictive coefficient $\widetilde{m}_{K}$ is also reduced by increasing porosity. On the other hand, Fig. 6(d) shows that the electrostrictive coefficient $\widetilde{m}_{J}$ is initially enhanced but subsequently reduced with the further addition of pores. As anticipated above, such variations of the effective material constants yield a direct competition of effects for the overall electrostriction capabilities of the composite.

Figure 7 shows the electrostriction ratios (63) for a porous dielectric elastomer with the same matrix and pore material properties as those considered in Fig. 6. Interestingly, the ratio of electrostrictions $\bar{H}_{11} / H_{11}^{m}$ transverse to the applied electric field is first slightly reduced $\left(\bar{H}_{11} / H_{11}^{m}<1\right)$ with the addition of pores before being enhanced $\left(\bar{H}_{11} / H_{11}^{m}>1\right)$ for porosities $c \geq 0.22$. On the other hand, the ratio of electrostrictions $\bar{H}_{33} / H_{33}^{m}$ in the direction of the applied electric field is significantly and monotonically enhanced $\left(\bar{H}_{33} / H_{33}^{m}>1\right)$. These results reveal that when isotropically adding vacuous spherical pores to (incompressible or) nearly incompressible dielectric elastomers, the reduction in stiffness dominates over the reduction in permittivity resulting in an enhancement of the electrostriction capabilities of these composite

\footnotetext{
${ }^{2}$ These values are typical of silicone rubber.
} 


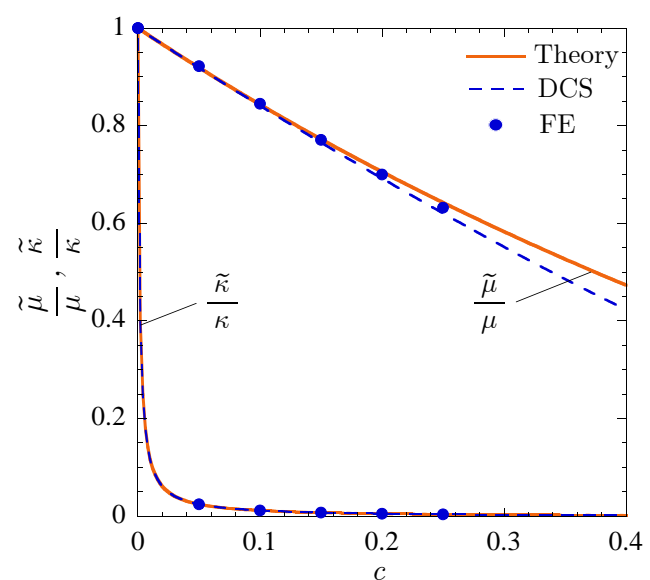

(a)

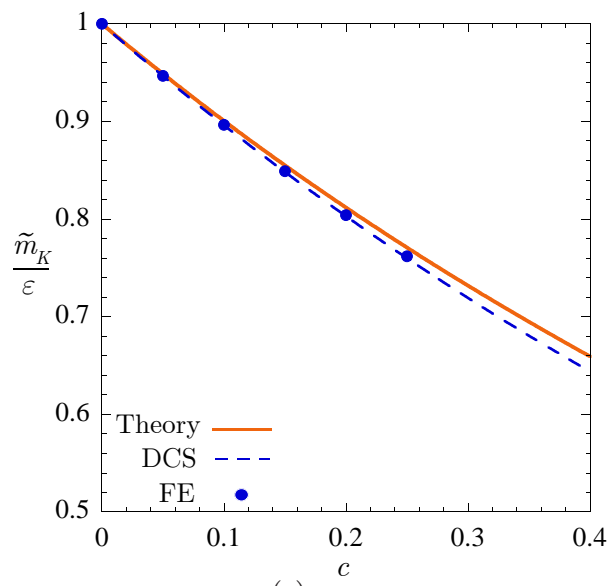

(c)

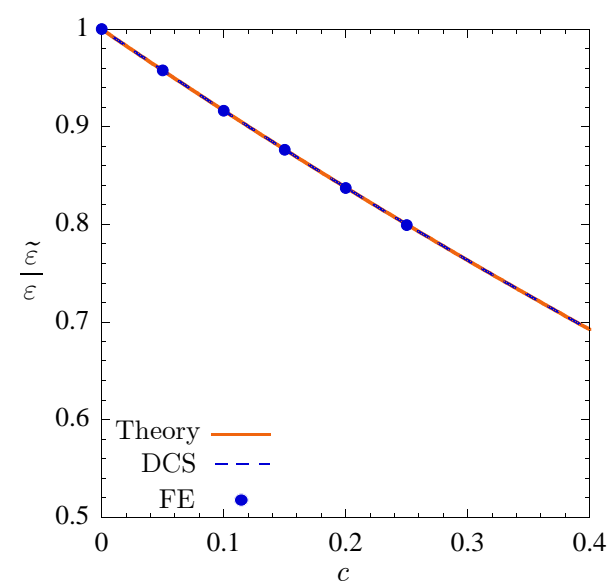

(b)

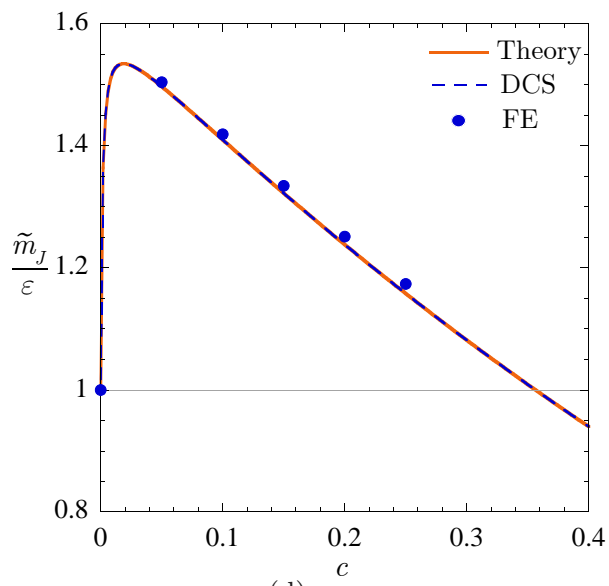

(d)

Figure 6: Overall electromechanical response of a nearly incompressible dielectric elastomer $\left(\kappa / \mu=10^{3}\right)$ with permittivity $\varepsilon=3 \varepsilon_{0}$ containing a random isotropic distribution of vacuous spherical pores $\left(\mu_{p}=0, \kappa_{p}=0, \varepsilon_{p}=\varepsilon_{0}\right)$. Results are shown for: (a) the normalized effective shear and bulk moduli $\widetilde{\mu} / \mu$ and $\widetilde{\kappa} / \kappa$, (b) the normalized effective permittivity $\widetilde{\varepsilon} / \varepsilon$, and (c)-(d) the normalized components of the effective electrostrictive tensor $\widetilde{m}_{K} / \varepsilon$ and $\widetilde{m}_{J} / \varepsilon$, all as functions of the volume fraction of pores c. The solid lines in the plots correspond to the theoretical results (49). The dashed lines and solid circles correspond to the analytical solution (64) for an isotropic suspension of polydisperse spherical particles and to FE simulations for an isotropic suspension of monodisperse spherical particles.

materials. Akin to the preceding results for dielectric elastomers filled with liquid-like high-permittivity particles, it would be interesting to explore these encouraging findings experimentally.

In summary, the above sample results have illustrated the capabilities of the general solutions (37), (38), (39) to provide quantitative insight into the overall electromechanical response of dielectric elastomer composites. They have also served to reveal that for the case of dielectric elastomers filled with a random isotropic distribution of stiff, high-permittivity or (semi-)conducting, roughly spherical particles — the case that has hitherto received most attention by the experimental community - interphasial phenomena may be crucial in understanding and exploiting the enhanced electrostriction that this promising class of materials are able to achieve. Furthermore, they have revealed that the study of dielectric elastomers filled with liquid-like high-permittivity or (semi-)conducting particles as well as porous dielectric elastomers may be worth pursuing. 


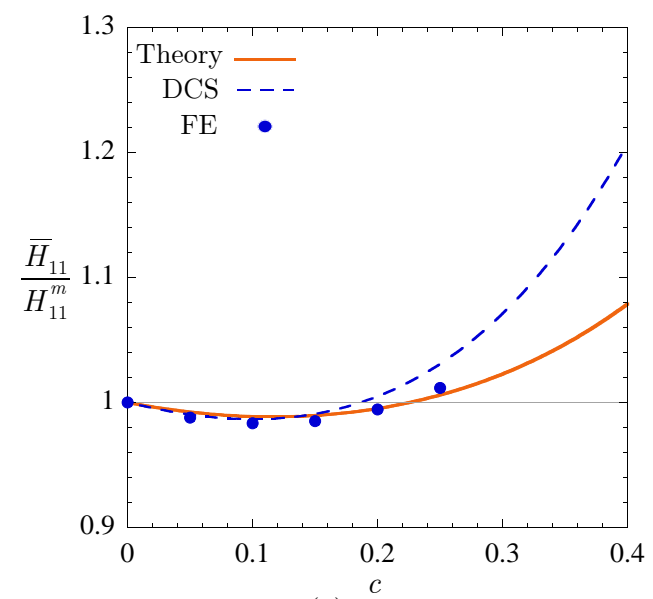

(a)

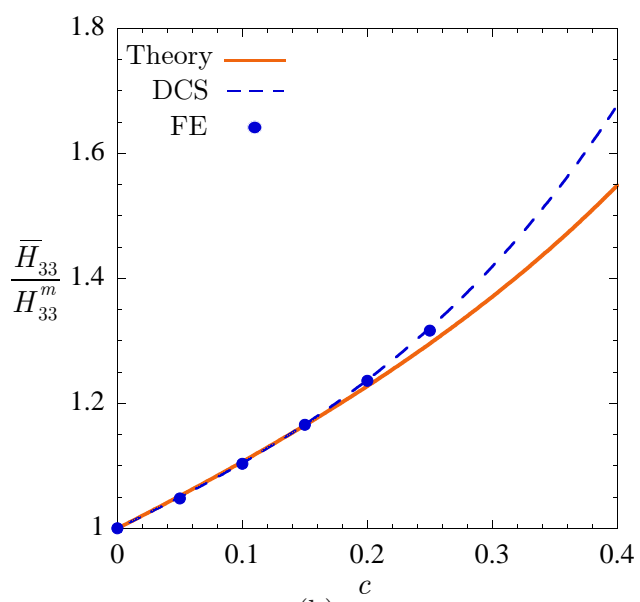

(b)

Figure 7: Electrostriction of a nearly incompressible dielectric elastomer $\left(\kappa / \mu=10^{3}\right)$ with permittivity $\varepsilon=3 \varepsilon_{0}$ containing a random isotropic distribution of vacuous spherical pores $\left(\mu_{p}=0, \kappa_{p}=0, \varepsilon_{p}=\varepsilon_{0}\right)$. Parts (a) and (b) show, respectively, the ratios of electrostrictions in the transverse and thickness directions, $\bar{H}_{11} / H_{11}^{m}$ and $\bar{H}_{33} / H_{33}^{m}$, as functions of the volume fraction of pores $c$. The solid lines in the plots correspond to expressions (63) based on the theoretical results (49). The dashed lines and solid circles stand for corresponding results based on the analytical solution (64) for an isotropic suspension of polydisperse spherical particles and on FE simulations for an isotropic suspension of monodisperse spherical particles.

\section{Acknowledgements}

Support for this work by the National Science Foundation through CAREER Grant CMMI-1219336 is gratefully acknowledged.

\section{Appendix A. FE calculations of the homogenized response of dielectric elastomer composites}

In this appendix, we summarize the computation of the finite-element (FE) results presented in Section 5 for dielectric elastomers filled with an isotropic distribution of spherical particles.

Within the classical context of small deformations and moderate electric fields described in Section 1.1, the effective electromechanical tensors $\widetilde{\mathbf{L}}, \widetilde{\boldsymbol{\epsilon}}, \widetilde{\mathbf{M}}$ for a composite material with arbitrarily heterogeneous local modulus of elasticity $\mathbf{L}=\mathbf{L}(\mathbf{X})$, permittivity $\boldsymbol{\epsilon}=\boldsymbol{\epsilon}(\mathbf{X})$, and electrostrictive tensor $\mathbf{M}=\mathbf{M}(\mathbf{X})$ are given by the formulas

$$
\begin{aligned}
& \widetilde{L}_{i j k l}=\frac{1}{|\Omega|} \int_{\Omega} L_{i j m n} \Gamma_{m k l, n} \mathrm{~d} \mathbf{X}, \\
& \widetilde{\epsilon}_{i j}=\frac{1}{|\Omega|} \int_{\Omega} \epsilon_{i m} \gamma_{m, j} \mathrm{~d} \mathbf{X}, \\
& \widetilde{M}_{i j k l}=\frac{1}{|\Omega|} \int_{\Omega} \Gamma_{m i j, n} M_{m n p q} \gamma_{p, k} \gamma_{q, l} \mathrm{~d} \mathbf{X} .
\end{aligned}
$$

Here, it is recalled that $\Omega$ stands for the domain occupied by the composite in its undeformed stress-free configuration and the tensor fields $\boldsymbol{\Gamma}$ and $\gamma$ are defined implicitly as the solutions to the following linear uncoupled boundary value problems:

$$
\left[L_{i j m n} \Gamma_{m k l, n}\right]_{, j}=0, \mathbf{X} \in \Omega \quad \text { with } \quad \Gamma_{i k l}=\delta_{i k} \delta_{j l} X_{j}, \mathbf{X} \in \partial \Omega,
$$




$$
\left[\epsilon_{i m} \gamma_{m, j}\right]_{, i}=0, \mathbf{X} \in \Omega \quad \text { with } \quad \gamma_{i}=\delta_{i j} X_{j}, \mathbf{X} \in \partial \Omega
$$

In the above expressions, the notation ${ }_{j}$ represents the partial differentiation with respect to the material point coordinate $X_{j}$ and $\partial \Omega$ stands for the boundary of the domain $\Omega$.

While the formulation (66)-(70) is formal in general, Tian (2007) and Tian et al. (2012) have shown it to be rigorous in the context of composite materials with periodic microstructure. When the repeating unit cell $Q$ chosen to describe such a periodic microstructure is the unit cube $Q=\left\{\mathbf{X}: X_{i} \in[0,1] \quad i=1,2,3\right\}$, the general formulation (66)-(70) specializes to

$$
\begin{aligned}
& \widetilde{L}_{i j k l}=\int_{Q} L_{i j m n} \Gamma_{m k l, n} \mathrm{~d} \mathbf{X}, \\
& \widetilde{\epsilon}_{i j}=\int_{Q} \epsilon_{i m} \gamma_{m, j} \mathrm{~d} \mathbf{X}, \\
& \widetilde{M}_{i j k l}=\int_{Q} \Gamma_{m i j, n} M_{m n p q} \gamma_{p, k} \gamma_{q, l} \mathrm{~d} \mathbf{X},
\end{aligned}
$$

where now the tensor fields $\boldsymbol{\Gamma}$ and $\gamma$ are defined by

$$
\begin{aligned}
& {\left[L_{i j m n} \Gamma_{m k l, n}\right]_{, j}=0, \mathbf{X} \in Q \quad \text { with } \quad\left\{\begin{array}{l}
\Gamma_{i k l}\left(1, X_{2}, X_{3}\right)-\Gamma_{i k l}\left(0, X_{2}, X_{3}\right)=\delta_{i k} \delta_{l 1} \\
\Gamma_{i k l}\left(X_{1}, 1, X_{3}\right)-\Gamma_{i k l}\left(X_{1}, 0, X_{3}\right)=\delta_{i k} \delta_{l 2}, \mathbf{X} \in \partial Q, \\
\Gamma_{i k l}\left(X_{1}, X_{2}, 1\right)-\Gamma_{i k l}\left(X_{1}, X_{2}, 0\right)=\delta_{i k} \delta_{l 3}
\end{array}\right.} \\
& {\left[\epsilon_{i m} \gamma_{m, j}\right]_{, i}=0, \mathbf{X} \in Q \quad \text { with }\left\{\begin{array}{l}
\gamma_{i}\left(1, X_{2}, X_{3}\right)-\gamma_{i}\left(0, X_{2}, X_{3}\right)=\delta_{i 1} \\
\gamma_{i}\left(X_{1}, 1, X_{3}\right)-\gamma_{i}\left(X_{1}, 0, X_{3}\right)=\delta_{i 2} \\
\gamma_{i}\left(X_{1}, X_{2}, 1\right)-\gamma_{i}\left(X_{1}, X_{2}, 0\right)=\delta_{i 3}
\end{array}\right.}
\end{aligned}
$$

with $\partial Q$ denoting the boundary of $Q$. It is plain that the partial differential equations (74)-(75), or more generally (69)-(70), do not admit explicit solutions other than for special cases. However, being secondorder linear elliptic pdes, they can be readily solved numerically by means of the finite element method, as outlined next.

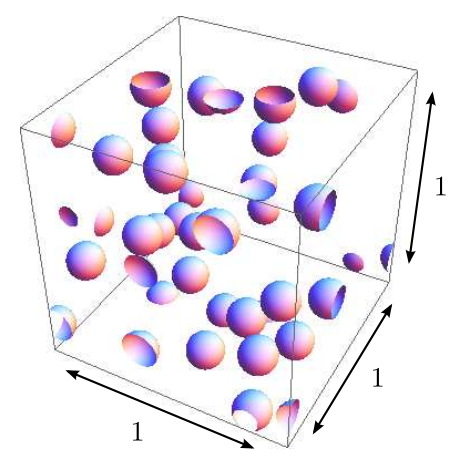

(a)

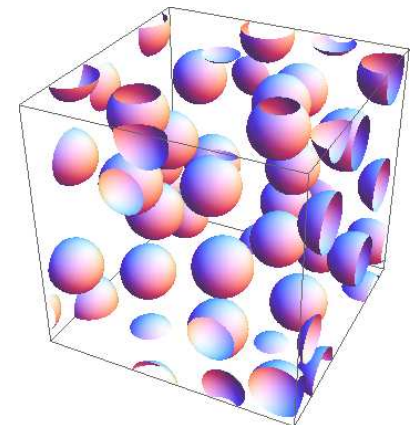

(b)

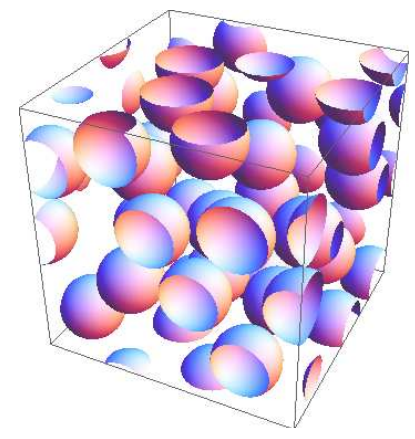

(c)

Figure 8: Unit cells with $N=30$ spherical particles randomly distributed for various particle concentrations: (a) $c=0.05$, (b) $c=0.15$, and (c) $c=0.25$. 
The focus of Section 5 is on the practical case of dielectric elastomers filled with a random and isotropic distribution of spherical particles. To approximate the randomness and isotropy of this class of microstructures, following common practice (see, e.g., Gusev, 1997; Michel et al., 1999), we begin by considering an infinite periodic medium where the repeated unit cell comprises a large but finite number $N$ of randomly distributed spherical particles. Previous results based on this approach - for linear (Segurado and Llorca, 2002) as well as for nonlinear (Lopez-Pamies et al., 2013b) problems - have indicated that $N=30$ particles is, in general, sufficient to approximate the isotropy of the microstructure. Figure 8 depicts representative examples of such unit cells with $N=30$ particles for various particle concentrations: (a) $c=0.05$, (b) $c=0.15$, and (c) $c=0.25$. Having identified the microstructures of interest, we carry out their discretizations

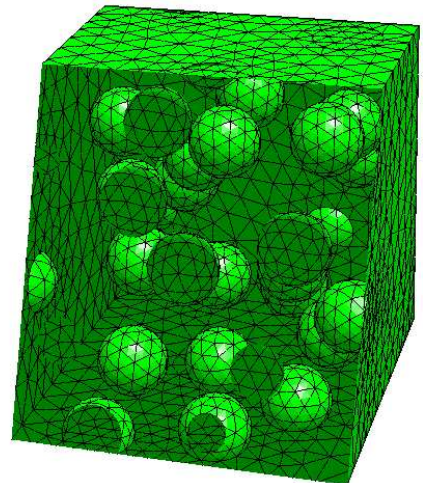

(a)

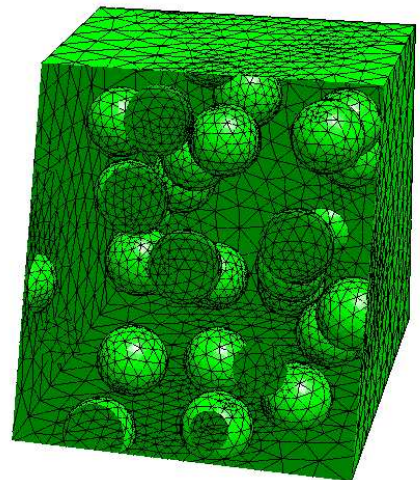

(b)

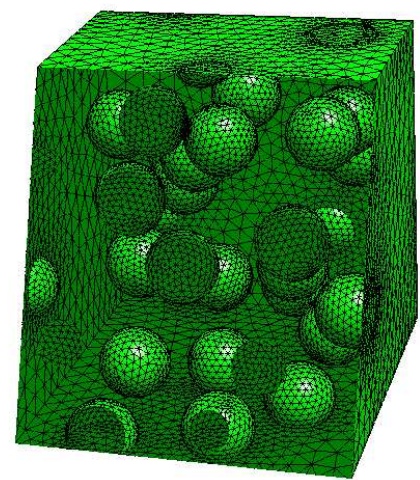

(c)

Figure 9: Three progressively refined meshes, with (a) 29,089, (b) 94,837, and (c) 416,544 elements, of a unit cell containing a random distribution of $N=30$ spherical particles at concentration $c=0.15$.

by means of the mesh generator code Netgen (Schöberl, 1997). Hybrid isoparametric 10-node tetrahedral elements with constant pressure were selected to solve the pde (74), while isoparametric 10-node tetrahedral elements were utilized for the pde (75). Figure 9 displays three meshes of increasing refinement with particle concentration $c=0.15$. Mesh sensitivity analyses revealed that meshes containing about 400,000 elements ( $\sim 570,000$ nodes $)$, such as the one shown in Fig. 9(c), are refined enough to deliver accurate results.

The resulting discretized version of equations (74)-(75) for $\Gamma$ and $\gamma$ are formulated and solved via a vectorized $\mathrm{FE}$ code written in the technical computing environment MATLAB (see MATLAB Version 8.3 Documentation, 2014). The computed FE solutions for the fields $\boldsymbol{\Gamma}$ and $\boldsymbol{\gamma}$ are then utilized in the definitions (71)-(73) to finally compute the three effective tensors that describe the macroscopic electromechanical response of the pertinent dielectric elastomer composite: $\widetilde{\mathbf{L}}^{F E}, \widetilde{\boldsymbol{\epsilon}}^{F E}$, and $\widetilde{\mathbf{M}}^{F E}$ (here and subsequently, for clarity, the superscript "FE" is appended to any quantity based on FE solutions).

Assessment of the simulated microstructures. Because of the finite number of particles $(N=30)$ included per unit cell, the microstructures simulated here are (not exactly but) only approximately isotropic. In order to assess the isotropy of each realization that is constructed, we monitor the following measures

$$
d_{\widetilde{\mathbf{L}}}=\frac{\left\|2 \widetilde{\mu}^{F E} \mathcal{K}+3 \widetilde{\kappa}^{F E} \mathcal{J}-\widetilde{\mathbf{L}}^{F E}\right\|_{\infty}}{\left\|\widetilde{\mathbf{L}}^{F E}\right\|_{\infty}}, \quad d_{\widetilde{\boldsymbol{\epsilon}}}=\frac{\left\|\widetilde{\varepsilon}^{F E} \mathbf{I}-\widetilde{\boldsymbol{\epsilon}}^{F E}\right\|_{\infty}}{\left\|\widetilde{\boldsymbol{\epsilon}}^{F E}\right\|_{\infty}}, \quad d_{\widetilde{\mathbf{M}}}=\frac{\left\|\widetilde{m}_{K}^{F E} \mathcal{K}+\widetilde{m}_{J}^{F E} \mathcal{J}-\widetilde{\mathbf{M}}^{F E}\right\|_{\infty}}{\left\|\widetilde{\mathbf{M}}^{F E}\right\|_{\infty}},
$$

where the effective electromechanical coefficients $\widetilde{\mu}^{F E}, \widetilde{\kappa}^{F E}, \widetilde{\varepsilon}^{F E}, \widetilde{m}_{K}^{F E}$, and $\widetilde{m}_{J}^{F E}$ are defined by

$$
\begin{aligned}
& \widetilde{\mu}^{F E} \doteq \frac{1}{10} \mathcal{K} \cdot \widetilde{\mathbf{L}}^{F E}=\frac{1}{20}\left(\widetilde{L}_{i j i j}^{F E}+\widetilde{L}_{i j j i}^{F E}-\frac{2}{3} \widetilde{L}_{i i j j}^{F E}\right), \\
& \widetilde{\kappa}^{F E} \doteq \frac{1}{3} \mathcal{J} \cdot \widetilde{\mathbf{L}}^{F E}=\frac{1}{9} \widetilde{L}_{i i j j}^{F E}
\end{aligned}
$$




$$
\begin{aligned}
& \widetilde{\varepsilon}^{F E} \doteq \frac{1}{3} \mathbf{I} \cdot \widetilde{\boldsymbol{\epsilon}}^{F E}=\frac{1}{3} \widetilde{\epsilon}_{i i}^{F E} \\
& \widetilde{m}_{K}^{F E} \doteq \frac{1}{5} \mathcal{K} \cdot \widetilde{\mathbf{M}}^{F E}=\frac{1}{10}\left(\widetilde{M}_{i j i j}^{F E}+\widetilde{M}_{i j j i}^{F E}-\frac{2}{3} \widetilde{M}_{i i j j}^{F E}\right) \\
& \widetilde{m}_{J}^{F E} \doteq \mathcal{J} \cdot \widetilde{\mathbf{M}}^{F E}=\frac{1}{3} \widetilde{M}_{i i j j}^{F E}
\end{aligned}
$$

and it is recalled that $\mathcal{K}$ and $\mathcal{J}$ stand for the orthogonal projection tensors given by expressions (28). For the class of dielectric elastomer composites of interest in Section 5, wherein the matrix and particles are both isotropic elastic dielectrics, a perfectly isotropic microstructure would result in measures $d_{\widetilde{\mathbf{L}}}=d_{\widetilde{\boldsymbol{\epsilon}}}=d_{\widetilde{\mathbf{M}}}=0$. In this work, only realizations with

$$
\max \left\{d_{\widetilde{\mathbf{L}}}, d_{\widetilde{\boldsymbol{\epsilon}}}, d_{\widetilde{\mathbf{M}}}\right\} \leq 10^{-2}
$$

are admitted as approximately isotropic.

We conclude by noting that all FE results presented in Section 5 correspond to the average of three different realizations (all satisfying the above definition of approximate isotropy). The effective electromechanical coefficients displayed in the plots correspond to the formulae (77)-(81), while the pertinent components of the electrostrictive deformation $\overline{\mathbf{H}}$ are based on the formulae

$$
\bar{H}_{11}^{F E}=\bar{H}_{22}^{F E}=\left(\frac{\widetilde{m}_{K}^{F E}}{6 \widetilde{\mu}^{F E}}-\frac{\widetilde{m}_{J}^{F E}}{9 \widetilde{\kappa}^{F E}}\right) \bar{E}^{2} \quad \text { and } \quad \bar{H}_{33}^{F E}=-\left(\frac{\widetilde{m}_{K}^{F E}}{3 \widetilde{\mu}^{F E}}+\frac{\widetilde{m}_{J}^{F E}}{9 \widetilde{\kappa}^{F E}}\right) \bar{E}^{2} .
$$

\section{References}

[1] Avellaneda, M., 1987. Iterated homogenization, differential effective medium theory and applications. Commun. Pur. Appl. Math. 40, 527-554.

[2] Bergman, D.J., 1978. The dielectric constant of a composite material-a problem in classical physics. Phys. Reports 43C, $377-407$.

[3] Carpi, F., De Rossi, D. 2005. Improvement of electromechanical actuating performances of a silicone dielectric elastomer by dispersion of titanium dioxide powder. IEEE Transactions on Dielectrics and Electrical Insulation 12, 835-843.

[4] Di Lillo, L., Schmidt, A., Bergamini, A., Ermanni, P., Mazza, E. 2011. Dielectric and insulating properties of an acrylic DEA material at high near-DC electric fields. Proc. of SPIE 7976, 79763B.

[5] Dorfmann, A., Ogden, R.W., 2005. Nonlinear electroelasticity. Acta Mechanica 174, 167-183.

[6] Eshelby, J.D., 1957. The determination of the elastic field of an ellipsoidal inclusion and related problems. Proc. R. Soc. London A 241, 376-396.

[7] Fassler, A., Majidi, C., 2015. Liquid-phase metal inclusions for a conductive polymer composite. Advanced Materials 27, $1928-1932$.

[8] Goudarzi, T., Spring, D.W., Paulino, G.H., Lopez-Pamies, O. 2015. Filled elastomers: A theory of filler reinforcement based on hydrodynamic and interphasial effects. J. Mech. Phys. Solids doi:10.1016/j.jmps.2015.04.012.

[9] Gusev, A.A., 1997. Representative volume element size for elastic composites: a numerical study. J. Mech. Phys. Solids $45,1449-1459$

[10] Hashin, Z., Shtrikman, S., 1962a. On some variational principles in anisotropic and nonhomogeneous elasticity. J. Mech. Phys. Solids 10, 335-342.

[11] Hashin, Z., Shtrikman, S., 1962b. A variational approach to the theory of the effective magnetic permeability of multiphase materials. Journal of Applied Physics 33, 3125-3131.

[12] Huang, C., Zhang, Q.M., 2004. Enhanced dielectric and electromechanical response in high-dielectric constant all-polymer percolative composites. Advanced Functional Materials 14, 501-506.

[13] Huang, C., Zhang, Q.M., Li, J.Y., Rabeony, M., 2005. Colossal dielectric and electromechanical responses in self-assembled polymeric nanocomposites. Applied Physics Letters 87, 182901.

[14] Kofod, G., Sommer-Larsen, P., Kornbluh, R., Pelrine, R., 2003. Actuation response of polyacrylate dielectric elastomers. J. Intell. Mater. Syst. Struct. 14, 787-793.

[15] Lewis, T.J., 2004. Interfaces are the dominant feature of dielectrics at the nanometric level. IEEE Transactions on Dielectrics and Electrical Insulation 11, 739-753.

[16] Lefèvre, V., Lopez-Pamies, O. 2014. The overall elastic dielectric properties of a suspension of spherical particles in rubber: An exact explicit solution in the small-deformation limit. Journal of Applied Physics 116, 134106.

[17] Li, J.Y., Rao, N., 2004. Micromechanics of ferroelectric polymer-based electrostrictive composites. J. Mech. Phys. Solids $52,591-615$. 
[18] Li, J.Y., Huang, C., Zhang, Q., 2004. Enhanced electromechanical properties in all-polymer percolative composites. Appl. Phys. Lett. 84, 3124.

[19] Liu, H., Zhang, L., Yang, D., Yu, Y., Yao, L., Tian, M. 2013. Mechanical, dielectric, and actuated strain of silicone elastomer filled with various types of $\mathrm{TiO}_{2}$. Soft Materials 11, 363-370.

[20] Lopez-Pamies, O., 2006. On the effective behavior, microstructure evolution, and macroscopic stability of elastomeric composites. Ph.D. Dissertation, University of Pennsylvania, USA.

[21] Lopez-Pamies, O., 2014. Elastic dielectric composites: Theory and application to particle-filled ideal dielectrics. J. Mech. Phys. Solids 64, 61-82.

[22] Lopez-Pamies, O., Goudarzi, T., Nakamura, T. 2013a. The nonlinear elastic response of suspensions of rigid inclusions in rubber: I - An exact result for dilute suspensions. J. Mech. Phys. Solids 61, 1-18.

[23] Lopez-Pamies, O., Goudarzi, T., Nakamura, T. 2013b. The nonlinear elastic response of suspensions of rigid inclusions in rubber: II - A simple explicit approximation for finite-concentration suspensions. J. Mech. Phys. Solids 61, $19-37$.

[24] Lopez-Pamies, O., Goudarzi, T., Meddeb , A.B., Ounaies, Z., 2014. Extreme enhancement and reduction of the dielectric response of polymer nanoparticulate composites via interphasial charges. Applied Physics Letters 104, 24290.

[25] MATLAB Version 8.3 Documentation, Mathworks, Natick, Massachusetts, USA, 2014.

[26] Mc Carthy, D.N., Risse, S., Katekomol, P., Kofod, G. 2009. The effect of dispersion on the increased relative permittivity of $\mathrm{TiO}_{2} / \mathrm{SEBS}$ composites. J. Phys. D: Appl. Phys. 42, 145406.

[27] Michel, J.C., Moulinec, H., Suquet, P. 1999. Effective properties of composite materials with periodic microstructure: a computational approach. Computer Methods in Applied Mechanics and Engineering 172, 109-143.

[28] Milton, G.W., 2002. The Theory of Composites. Cambridge Monographs on Applied and Computational Mathematics, Cambridge University Press, Cambridge, Vol. 6.

[29] Roy, M., Nelson, J.K., MacCrone, R.K., Schadler, L.S., Reed, C.W., Keefe, R., Zenger, W., 2005. Polymer nanocomposite dielectrics - The role of the interface. IEEE Transactions on Dielectrics and Electrical Insulation 12, 629-643.

[30] Schöberl, J. 1997. Netgen an advancing front 2d/3d-mesh generator based on abstract rules. Computing and Visualization in Science 1, 41-52.

[31] Segurado, J., Llorca, J. 2002. A numerical approximation to the elastic properties of sphere-reinforced composites. J. Mech. Phys. Solids 50, 2107-2121.

[32] Siboni, M.H., Ponte Castañeda, P., 2013. Dielectric elastomer composites: small-deformation theory and applications. Philosophical Magazine 93, 2769-2801.

[33] Spinelli, S.A., Lopez-Pamies, O., 2014. A general closed-form solution for the overall response of piezoelectric composites with periodic and random particulate microstructures. Int. J. Solids Struct. 51, 2979-2989.

[34] Stratton, J.S., 1941. Electromagnetic Theory. McGraw-Hill.

[35] Suo, Z., Zhao, X., Greene, W.H., 2008. A nonlinear field theory of deformable dielectrics. J. Mech. Phys. Solids 56, $467-486$.

[36] Tian, L., 2007. Effective Behavior of Dielectric Elastomer Composites. Ph.D. Thesis, California Institute of Technology.

[37] Tian, L., Tevet-Deree, L., deBotton, G., Bhattacharya, K., 2012. Dielectric elastomer composites. J. Mech. Phys. Solids 60, 181-198.

[38] Toupin, R.A., 1956. The elastic dielectric. J. Ration. Mech. Anal. 5, 849-915.

[39] Wang, Q., Suo, Z., Zhao, X., 2012. Bursting drops in solid dielectrics caused by high voltages. Nature Communications $3,1157$.

[40] Willis, J.R., 1977. Bounds and self-consistent estimates for the overall moduli of anisotropic composites. J. Mech. Phys. Solids 25, 185-202.

[41] Wissler, M., Mazza, E., 2007. Electromechanical coupling in dielectric elastomer actuators. Sensors and Actuators A 138, 384-393.

[42] Zhang, Q.M., Hengfeng, L., Poh, M., Xia, F., Cheng, Z.-Y., Xu, H., Huang, C., 2002. An all-organic composite actuator material with high dielectric constant. Nature 419, 284-287. 